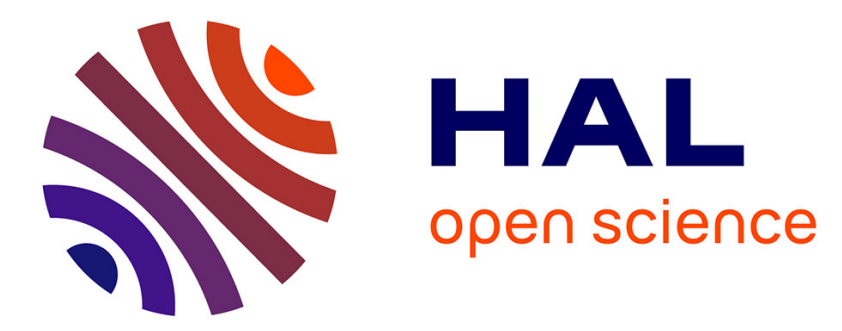

\title{
Self-similar random fields and rescaled random balls models
}

\author{
Hermine Biermé, Anne Estrade, Ingemar Kaj
}

\section{To cite this version:}

Hermine Biermé, Anne Estrade, Ingemar Kaj. Self-similar random fields and rescaled random balls models. Journal of Theoretical Probability, 2010, 23 (4), pp.1110-1141. hal-00161614v2

\section{HAL Id: hal-00161614 \\ https://hal.science/hal-00161614v2}

Submitted on 12 Feb 2008

HAL is a multi-disciplinary open access archive for the deposit and dissemination of scientific research documents, whether they are published or not. The documents may come from teaching and research institutions in France or abroad, or from public or private research centers.
L'archive ouverte pluridisciplinaire HAL, est destinée au dépôt et à la diffusion de documents scientifiques de niveau recherche, publiés ou non, émanant des établissements d'enseignement et de recherche français ou étrangers, des laboratoires publics ou privés. 


\title{
SELF-SIMILAR RANDOM FIELDS AND RESCALED RANDOM BALLS MODELS
}

\author{
HERMINE BIERMÉ, ANNE ESTRADE, AND INGEMAR KAJ
}

\begin{abstract}
We study generalized random fields which arise as rescaling limits of spatial configurations of uniformly scattered random balls as the mean radius of the balls tends to 0 or infinity. Assuming that the radius distribution has a power law behavior, we prove that the centered and re-normalized random balls field admits a limit with spatial dependence and self-similarity properties. In particular, our approach provides a unified framework to obtain all self-similar, translation and rotation invariant Gaussian fields. Under specific assumptions, we also get a Poisson type asymptotic field. In addition to investigating stationarity and self-similarity properties, we give L2-representations of the asymptotic generalized random fields viewed as continuous random linear functionals.
\end{abstract}

\section{INTRODUCTION}

In this work we construct essentially all Gaussian, translation and rotation invariant, $H$-self-similar generalized random fields on $\mathbb{R}^{d}$ in a unified manner as scaling limits of a random balls model. The self-similarity index $H$ ranges over all of $\mathbb{R} \backslash \mathbb{Z}$ and the random balls model is of germ-grain type. It arises by aggregation of spherical grains attached to uniformly scattered germs given by a Poisson point process in $d$-dimensional space. By a similar scaling procedure, we obtain also non-Gaussian random fields with interesting properties, in particular a model of the type "fractional Poisson field". Its covariance functional coincides with that of the Gaussian $H$-self-similar field, so that it fulfills a second order self-similarity property. Although not self-similar in law, this Poisson field presents a property of "aggregate similarity" which takes into account both Poisson structure and self-similarity.

We observe two distinctly separate behaviors depending on whether the self-similarity index $H$ belongs to an interval of type $(m, m+1 / 2)$ or of type $[m-1 / 2, m)$ for some integer $m$. In the first case, the scaling limit applies to random balls models with balls of arbitrarily small radii. The asymptotic field then provides spatial dependence of negative type. In the opposite case, the corresponding germ-grain models have arbitrarily large spherical grains which lead to spatial long range dependence.

The scaling procedure which acts on the random balls model is based on the assumption that the grains have random radius, independent and identically distributed, with a distribution having a power law behavior either in zero or at infinity. The resulting

2000 Mathematics Subject Classification. Primary: 60G60, 60G78; Secondary: 60G20, 60D05, 60G55, 60G10, 60F05.

Keywords: self-similarity, generalized random field, Poisson point process, fractional Poisson field, fractional Brownian field.

Date: February 12, 2008.

This work was supported by ANR grant "mipomodim" ANR-05-BLAN-0017. 
configuration of mass, obtained by counting the number of balls that cover any given point in space, suitably centered and normalized, exhibits limit distributions under scaling. For the case of the random balls radius distribution being heavy-tailed at infinity, the corresponding scaling operation amounts to zooming out over larger areas of space while re-normalizing the mass. In the opposite case, when the radius of balls is given by an intensity with prescribed power-law behavior close to zero, the scaling which is applied entails zooming in successively smaller regions of space. Infinitesimally small microballs will emerge and eventually shape the resulting limit fields.

Our results unify and extend in some directions the previous works on similar topics in Kaj et al. [13] and Biermé and Estrade [4]. For example, in terms of the self-similarity index $H,[13$ ] covers the interval $-d / 2<H<0$ and [4] the interval $0<H<1 / 2$. Preliminary and less general versions of some of the results presented here have appeared in Biermé et al. [5].

Dobrushin [7] characterized the stationary self-similar Gaussian generalized random fields in their spectral form. In this work we obtain the subclass of such random fields that are isotropic, since the random balls models under consideration are rotationally symmetric. In order to obtain the whole range of self-similarity behavior it is necessary to work not only with stationary random fields but with the wider class of generalized random fields with stationary increments or stationary $n$th increments. In this sense our approach also links to the line of work initiated by Matheron [16].

The paper is organized as follows. After having introduced the modeling framework and the setting of the investigation we discuss in Section 2 some principles for scaling limit analysis and state two main results, which cover a Gaussian limit regime and a Poisson limit regime. Section 3 is devoted to the study of all self-similar, isotropic, stationary generalized random fields. In particular, our main result states that all such Gaussian fields arise as scaling limits of the random balls model. In Section 4 we give a pointwise representation of the generalized self-similar fields with positive self-similarity index $H>0$ and discuss a few explicit examples. We conclude with some further remarks on aggregate similarity.

\section{Setting}

We present first a unified framework which includes and extends both of the distinct modeling scenarios studied in [13] and [4], respectively. Let $B(x, r)$ denote the ball in $\mathbb{R}^{d}$ with center at $x$ and radius $r$ and consider a family of grains $X_{j}+B\left(0, R_{j}\right)$ in $\mathbb{R}^{d}$ generated by a Poisson point process $\left(X_{j}, R_{j}\right)_{j}$ in $\mathbb{R}^{d} \times \mathbb{R}^{+}$. Equivalently, we let $N(\mathrm{~d} x, \mathrm{~d} r)$ be a Poisson random measure on $\mathbb{R}^{d} \times \mathbb{R}^{+}$and associate with each random point $(x, r) \in \mathbb{R}^{d} \times \mathbb{R}^{+}$the random ball $B(x, r)$. We assume that the intensity measure of $N$ is given by $\mathrm{d} x F(\mathrm{~d} r)$ where $F$ is a $\sigma$-finite non-negative measure on $\mathbb{R}^{+}$. Moreover, we assume throughout the paper that the ball radius intensity $F(\mathrm{~d} r)$ is such that

$$
\int_{\mathbb{R}^{+}} r^{d} F(\mathrm{~d} r)<+\infty .
$$

Note that if $F$ is a probability measure, this assumption implies that the expected balls volume is finite. 
For measurable sets $A \subset \mathbb{R}^{d} \times \mathbb{R}^{+}$we let the map $A \mapsto N(A)$ count the number of balls subject to $A$ with values viewed as real-valued random variables on a probability space $(\Omega, \mathcal{A}, \mathbb{P})$. We recall the basic facts that $N(A)$ is Poisson distributed with mean $\int_{A} \mathrm{~d} x F(\mathrm{~d} r)$ (if the integral diverges then $N(A)$ is countably infinite with probability one) and if $A_{1}, \ldots, A_{n}$ are disjoint then $N\left(A_{1}\right), \ldots, N\left(A_{n}\right)$ are independent. We also recall that for measurable functions $k: \mathbb{R}^{d} \times \mathbb{R}^{+} \rightarrow \mathbb{R}$, the stochastic integral $\int k(x, r) N(\mathrm{~d} x, \mathrm{~d} r)$ of $k$ with respect to $N$ exists $\mathbb{P}$-a.s. if and only if

$$
\int_{\mathbb{R}^{d} \times \mathbb{R}^{+}} \min (|k(x, r)|, 1) \mathrm{d} x F(\mathrm{~d} r)<\infty .
$$

1.1. Power-law assumption. For $\beta \neq d$ we introduce the following asymptotic power law assumption for the behavior of $F$ near 0 or at infinity:

$$
\mathbf{A}(\beta): \quad F(\mathrm{~d} r)=f(r) \mathrm{d} r \text { with } f(r) \sim C_{\beta} r^{-\beta-1}, \text { as } r \rightarrow 0^{d-\beta},
$$

where $C_{\beta}>0$ and by convention $0^{\alpha}=0$ if $\alpha>0$ and $0^{\alpha}=+\infty$ if $\alpha<0$.

The range of parameter values under consideration will be $d-1<\beta<2 d$. Then, according to (1), under assumption $\mathbf{A}(\beta)$ it is natural to consider the asymptotic behavior of $F$ near 0 for $d-1<\beta<d$ and at infinity for $d<\beta<2 d$.

1.2. Random field. We consider random fields defined on a space of measures, in the same spirit as the random functionals of [13] or the generalized random fields of [3]. Let $\mathcal{M}$ denote the space of signed measures $\mu$ on $\mathbb{R}^{d}$ with finite total variation $|\mu|\left(\mathbb{R}^{d}\right)<\infty$, where $|\mu|$ is the total variation measure of $\mu$. For any $\mu \in \mathcal{M}, \mu(B(x, r))$ is a measurable function on $\mathbb{R}^{d} \times \mathbb{R}^{+}$for which

$$
\int_{\mathbb{R}^{d} \times \mathbb{R}^{+}}|\mu(B(x, r))| \mathrm{d} x F(\mathrm{~d} r) \leq|B(0,1)||\mu|\left(\mathbb{R}^{d}\right) \int_{\mathbb{R}^{+}} r^{d} F(\mathrm{~d} r)<+\infty,
$$

in view of (1). In particular, (2) applies for this choice of $k$. We may hence introduce a generalized random field $X$ defined on $\mathcal{M}$ by

$$
X(\mu)=\int_{\mathbb{R}^{d} \times \mathbb{R}^{+}} \mu(B(x, r)) N(\mathrm{~d} x, \mathrm{~d} r), \forall \mu \in \mathcal{M} .
$$

The condition (3) is even sufficient and necessary for $X(\mu)$ to have finite expected value, and in this case

$$
\mathbb{E} X(\mu)=\int_{\mathbb{R}^{d} \times \mathbb{R}^{+}} \mu(B(x, r)) \mathrm{d} x F(\mathrm{~d} r)=c_{d} \mu\left(\mathbb{R}^{d}\right) \int_{\mathbb{R}^{+}} r^{d} F(\mathrm{~d} r),
$$

where $c_{d}=|B(0,1)|$ is the volume of the unit ball in $\mathbb{R}^{d}$. Let us also note that the random field $X$ is linear on each vectorial subspace of $\mathcal{M}$ in the sense that for all $\mu_{1}, \ldots, \mu_{n} \in \mathcal{M}$ and $a_{1}, \ldots, a_{n} \in \mathbb{R}$, almost surely,

$$
X\left(a_{1} \mu_{1}+\ldots+a_{n} \mu_{n}\right)=a_{1} X\left(\mu_{1}\right)+\ldots+a_{n} X\left(\mu_{n}\right) .
$$

Our first proposition adds to this a simple topological structure.

Proposition 1.1. The random field $X$ is a continuous random linear functional on $\mathcal{M}$. 
Proof. It is natural to consider the weak topology on $\mathcal{M}$ where the weak convergence $\mu_{n} \rightarrow \mu$ is equivalent to

$$
\int_{\mathbb{R}^{d}} f(z) \mu_{n}(\mathrm{~d} z) \rightarrow \int_{\mathbb{R}^{d}} f(z) \mu(\mathrm{d} z),
$$

for all $f \in \mathcal{C}_{b}\left(\mathbb{R}^{d}\right)$ the space of continuous and bounded functions on $\mathbb{R}^{d}$. A sequence of signed measures $\left(\mu_{n}\right)_{n \in \mathbb{N}}$ converges weakly to $\mu \in \mathcal{M}$ if and only if the sequence of its total variations $\left(\left|\mu_{n}\right|\right)_{n \in \mathbb{N}}$ converges weakly to $|\mu|$ in $\mathcal{M}$. This is a consequence of the Radon Nikodym decomposition theorem (see [19] for instance). Then, in particular, if $\mu_{n} \rightarrow 0$ we have $\left|\mu_{n}\right|\left(\mathbb{R}^{d}\right) \rightarrow 0$.

Now, for any $\mu \in \mathcal{M}$ the random variable $X(\mu)$ is in $L^{2}(\Omega, \mathcal{A}, \mathbb{P})$ and so $X$ can be considered as a linear functional $X: \mathcal{M} \rightarrow L^{2}(\Omega, \mathcal{A}, \mathbb{P})$. Indeed, for any $\mu \in \mathcal{M}$

$$
\begin{aligned}
\operatorname{Var}(X(\mu)) & =\int_{\mathbb{R}^{d} \times \mathbb{R}^{+}} \mu(B(x, r))^{2} \mathrm{~d} x F(\mathrm{~d} r) \\
& =\int_{\mathbb{R}^{d} \times \mathbb{R}^{d}} \mu(\mathrm{d} y) \mu\left(\mathrm{d} y^{\prime}\right) \int_{\mathbb{R}^{+}}\left|B(y, r) \cap B\left(y^{\prime}, r\right)\right| F(\mathrm{~d} r) \\
& \leq c_{d}\left(\int_{\mathbb{R}^{+}} r^{d} F(\mathrm{~d} r)\right)|\mu|\left(\mathbb{R}^{d}\right)^{2}<\infty .
\end{aligned}
$$

Similarly, $|\mathbb{E}(X(\mu))| \leq c_{d}\left(\int_{0}^{+\infty} r^{d} F(\mathrm{~d} r)\right)|\mu|\left(\mathbb{R}^{d}\right)$. Then $X$ is continuous since $\mu_{n} \rightarrow 0$ in $\mathcal{M}$ implies that $\mathbb{E}\left(X\left(\mu_{n}\right)^{2}\right) \rightarrow 0$.

The random linear functional $X-\mathbb{E}(X)$ is also continuous from $\mathcal{M}$ to $L^{2}(\Omega, \mathcal{A}, \mathbb{P})$. Moreover, by identifying the space $\mathcal{M}$ with $\mathcal{C}_{0}\left(\mathbb{R}^{d}\right)^{\prime}$, the topological dual space of continuous functions that tend to 0 at infinity (see e.g. [19] p.161), which is a normed space, we can consider the subordinated norm of $X-\mathbb{E}(X)$. For $\mu=\delta_{0}$, the Dirac mass at the origin of $\mathbb{R}^{d}$, we get $\operatorname{Var}\left(X\left(\delta_{0}\right)\right)=c_{d}\left(\int_{\mathbb{R}^{+}} r^{d} F(\mathrm{~d} r)\right)$ and may conclude that

$$
\|X-\mathbb{E}(X)\|=\sqrt{\left(c_{d} \int_{\mathbb{R}^{+}} r^{d} F(\mathrm{~d} r)\right)} .
$$

\section{SCALing Limit}

2.1. Scaled random fields. Let us introduce now the notion of "scaling", by which we indicate an action: a change of scale acts on the size of the grains. The scaling procedure performed in [13] acts on grains of volume $v$ changed by shrinking into grains of volume $\rho v$ with a small parameter $\rho$ ("small scaling" behavior). The same is performed in [4] in the context of a homogenization, but the scaling acts in the opposite way: the radii $r$ of grains are changed into $r / \varepsilon$ (which is a "large scaling" behavior). To cover both mechanisms we introduce the random field which is obtained by applying the rescaling of measures $\mu \mapsto \mu^{\rho}$, where $\mu^{\rho}(B)=\mu(\rho B)$ for $\rho>0$ and measurable subsets $B$ of $\mathbb{R}^{d}$. Let us denote by $F_{\rho}(\mathrm{d} r)$ the image measure of $F(\mathrm{~d} r)$ by the change of scale $r \mapsto \rho r$ and remark that

$$
X\left(\mu^{\rho}\right)=\int_{\mathbb{R}^{d} \times \mathbb{R}^{+}} \mu(B(x, r)) N\left(\mathrm{~d} \rho^{-1} x, \mathrm{~d} \rho^{-1} r\right), \forall \mu \in \mathcal{M},
$$


where the intensity measure of $N\left(\mathrm{~d} \rho^{-1} x, \mathrm{~d} \rho^{-1} r\right)$ is $\rho^{-d} \mathrm{~d} x F_{\rho}(\mathrm{d} r)$. It is natural from this viewpoint to have $\mu$ representing an observation window and interpret limits $\rho \rightarrow 0$ as zoom-out and limits $\rho \rightarrow \infty$ as zoom-in of the random configurations of balls in space. We will see that during zoom-out the appearance of occasional balls of very large radius provide enough overlap between balls to create strong positive dependence (the case $\beta>d$ ), while zooming in among microballs will generate in the asymptotic limit negatively dependent spatial random fields (the case $\beta<d$ ).

Let us multiply the intensity measure by $\lambda>0$ and consider the associated random field on $\mathcal{M}$ given by

$$
\int_{\mathbb{R}^{d} \times \mathbb{R}^{+}} \mu(B(x, r)) N_{\lambda, \rho}(\mathrm{d} x, \mathrm{~d} r)
$$

where $N_{\lambda, \rho}(\mathrm{d} x, \mathrm{~d} r)$ is the Poisson random measure with intensity measure $\lambda \mathrm{d} x F_{\rho}(\mathrm{d} r)$ and $\mu \in \mathcal{M}$. Results are expected concerning the asymptotic behavior of this scaled random balls model under hypothesis $\mathbf{A}(\beta)$ when $\rho \rightarrow 0$ or $\rho \rightarrow+\infty$. We choose $\rho$ as the basic model parameter, consider $\lambda=\lambda(\rho)$ as a function of $\rho$, and define on $\mathcal{M}$ the random field

$$
X_{\rho}(\mu)=\int_{\mathbb{R}^{d} \times \mathbb{R}^{+}} \mu(B(x, r)) N_{\lambda(\rho), \rho}(\mathrm{d} x, \mathrm{~d} r) .
$$

Then, we are looking for a normalization term $n(\rho)$ such that the centered field converges in distribution,

$$
\frac{X_{\rho}(.)-\mathbb{E}\left(X_{\rho}(.)\right)}{n(\rho)} \stackrel{f d d}{\rightarrow} W(.)
$$

and we are interested in the nature of the limit field $W$. The convergence (7) holds whenever

$$
\mathbb{E}\left(\exp \left(i \frac{X_{\rho}(\mu)-\mathbb{E}\left(X_{\rho}(\mu)\right)}{n(\rho)}\right)\right) \rightarrow \mathbb{E}(\exp (i W(\mu))),
$$

for all $\mu$ in a convenient subspace of $\mathcal{M}$. A scaling analysis of power law tails reveals that under $\mathbf{A}(\beta)$ we expect

$$
\operatorname{Var}\left(X_{\rho}(\mu)\right) \sim \lambda(\rho) \rho^{\beta} \operatorname{Var}(X(\mu)), \quad \rho^{d-\beta} \rightarrow 0,
$$

which suggests the asymptotic relation $n(\rho)^{2} \sim \lambda(\rho) \rho^{\beta}$. However, in view of (5), the norm of $\left(X_{\rho}-\mathbb{E}\left(X_{\rho}\right)\right) / n(\rho)$ as a linear functional from $\mathcal{M}$ to $L^{2}(\Omega, \mathcal{A}, \mathbb{P})$ is given by

$$
\left\|\frac{X_{\rho}-\mathbb{E}\left(X_{\rho}\right)}{n(\rho)}\right\|=\sqrt{\left(c_{d} \int_{\mathbb{R}^{+}} r^{d} F(\mathrm{~d} r)\right)} \sqrt{\frac{\lambda(\rho) \rho^{d}}{n(\rho)^{2}}} .
$$

In particular, (8) is not bounded for $n(\rho)^{2}=\lambda(\rho) \rho^{\beta}$ as $\rho \rightarrow 0^{\beta-d}$ and the Banach Steinhaus Theorem states that there exists a dense subset of $\mathcal{M}$ on which the rescaled process $\left(X_{\rho}(\mu)-\mathbb{E}\left(X_{\rho}(\mu)\right)\right) / \sqrt{\lambda(\rho) \rho^{\beta}}$ can not converge. Therefore we study in the sequel the convergence $(7)$ on strict subspaces of $\mathcal{M}$. 
2.2. Gaussian limit regime. For $\beta \neq d$ let us define the space of measures

$$
\begin{aligned}
\mathcal{M}^{\beta}=\{\mu \in \mathcal{M}: & \exists \alpha \text { s.t. } \alpha<\beta<d \text { or } d<\beta<\alpha \\
& \text { and } \left.\int_{\mathbb{R}^{d} \times \mathbb{R}^{d}}\left|z-z^{\prime}\right|^{d-\alpha}|\mu|(\mathrm{d} z)|\mu|\left(\mathrm{d} z^{\prime}\right)<+\infty\right\} .
\end{aligned}
$$

We remark that the integral assumption is a finite Riesz energy assumption for $\beta>d$ and that $\mathcal{M}^{\beta}=\{0\}$ when $\beta \geq 2 d$. In both cases $d-1<\beta<d$ and $d<\beta<2 d$, if $\mu \in \mathcal{M}$ satisfies

$$
\int_{\mathbb{R}^{d} \times \mathbb{R}^{d}}\left|z-z^{\prime}\right|^{d-\alpha}|\mu|(\mathrm{d} z)|\mu|\left(\mathrm{d} z^{\prime}\right)<+\infty
$$

for some $\alpha$ ( $\alpha<\beta<d$ and $d<\beta<\alpha$ respectively) then the same holds for any $\gamma$ between $\beta$ and $\alpha$. In particular, for any $\mu \in \mathcal{M}^{\beta}$,

$$
\int_{\mathbb{R}^{d} \times \mathbb{R}^{d}}\left|z-z^{\prime}\right|^{d-\beta}|\mu|(\mathrm{d} z)|\mu|\left(\mathrm{d} z^{\prime}\right)<+\infty .
$$

We also introduce the subspace of finite signed measures of vanishing total mass,

$$
\mathcal{M}_{1}=\left\{\mu \in \mathcal{M}: \int_{\mathbb{R}^{d}} \mu(\mathrm{d} z)=0\right\}
$$

and consider the subspaces

$$
\widetilde{\mathcal{M}}_{\beta}= \begin{cases}\mathcal{M}^{\beta} & \text { for } d<\beta<2 d \\ \mathcal{M}^{\beta} \cap \mathcal{M}_{1} & \text { for } d-1<\beta<d .\end{cases}
$$

Theorem 2.1. Let $d-1<\beta<2 d$ with $\beta \neq d$. Let $F$ be a non-negative measure on $\mathbb{R}^{+}$ which satisfies $\mathbf{A}(\beta)$. For all positive functions $\lambda$ such that $\lambda(\rho) \rho^{\beta} \underset{\rho \rightarrow 0^{\beta-d}}{\longrightarrow}+\infty$, the limit

$$
\frac{X_{\rho}(\mu)-\mathbb{E}\left(X_{\rho}(\mu)\right)}{\sqrt{\lambda(\rho) \rho^{\beta}}} \underset{\rho \rightarrow 0^{\beta-d}}{\stackrel{f d d}{\longrightarrow}} W_{\beta}(\mu)
$$

holds for all $\mu \in \widetilde{\mathcal{M}}_{\beta}$, in the sense of finite dimensional distributions of the random functionals. Here $W_{\beta}$ is the centered Gaussian random linear functional on $\widetilde{\mathcal{M}}_{\beta}$ with covariance functional

$$
\operatorname{Cov}\left(W_{\beta}(\mu), W_{\beta}(\nu)\right)=\mathbb{E}\left(W_{\beta}(\mu) W_{\beta}(\nu)\right)=c_{\beta} \int_{\mathbb{R}^{d} \times \mathbb{R}^{d}}\left|z-z^{\prime}\right|^{d-\beta} \mu(\mathrm{d} z) \nu\left(\mathrm{d} z^{\prime}\right),
$$

for a constant $c_{\beta}$ depending on $\beta$.

Proof. We begin with two lemmas. The first lemma describes the covariance function and is based on some technical estimates for the intersection volume of two balls. The second one, inspired by Lemma 1 of [13], stands for Lebesgue's Theorem with assumptions that are well adapted to the present setting.

Lemma 2.2. Let $d-1<\beta<2 d$ with $\beta \neq d$. With $C_{\beta}>0$ given in $\mathbf{A}(\beta)$, there exists a real constant $c_{\beta}$ such that for all $\mu \in \widetilde{\mathcal{M}}_{\beta}$,

$$
0<C_{\beta} \int_{\mathbb{R}^{d} \times \mathbb{R}^{+}} \mu(B(x, r)) 2 r^{-\beta-1} \mathrm{~d} r \mathrm{~d} x=c_{\beta} \int_{\mathbb{R}^{d} \times \mathbb{R}^{d}}\left|z-z^{\prime}\right|^{d-\beta} \mu(\mathrm{d} z) \mu\left(\mathrm{d} z^{\prime}\right)<+\infty .
$$


Remark 2.3. The above identity ensures that equation (10) defines a covariance function, called generalized covariance function in [16].

Proof. Let us introduce the function $\gamma$ defined on $[0, \infty)$ by

$$
\gamma(u)=|B(0,1) \cap B(u \mathbf{e}, 1)|,
$$

for any unit vector $\mathbf{e} \in \mathbb{R}^{d}$, where $|\cdot|$ is the Lebesgue measure. The function $\gamma$ is decreasing, supported on $[0,2]$, bounded by $\gamma(0)=|B(0,1)|$, continuous on $[0,2]$, and smooth on $(0,2)$.

Define $\gamma_{\beta}$ as

$$
\gamma_{\beta}(u)= \begin{cases}\gamma(u)-\gamma(0), & d-1<\beta<d \\ \gamma(u), & d<\beta<2 d\end{cases}
$$

We notice that for $d-1<\beta<d,\left|\gamma_{\beta}(u)\right| \leq \gamma(0)$ as well as $\left|\gamma_{\beta}(u)\right| \leq \sup _{v>0}\left|\gamma^{\prime}(v)\right| u$. Hence, for some constant $C>0,\left|\gamma_{\beta}(u)\right| \leq C u^{d-\alpha}$ for any $0 \leq d-\alpha \leq 1$, that is any $\alpha$ in $[d-1, d]$. For $d<\beta<2 d$, one can find $C>0$ such that $\left|\gamma_{\beta}(u)\right| \leq C u^{d-\alpha}$ for any $\alpha \geq \beta$. In particular, we may take $\alpha$ such that $d-1<\alpha<\beta$ for the case $d-1<\beta<d$ and $\alpha$ such that $\beta<\alpha<2 d$ for $d<\beta<2 d$, and for both cases have a $C>0$ with

$$
\forall u>0,\left|\gamma_{\beta}(u)\right| \leq C u^{d-\alpha} .
$$

1st Step. For $\mu \in \widetilde{\mathcal{M}}_{\beta}$, let us prove that $\int_{\mathbb{R}^{d} \times \mathbb{R}^{+}} \mu(B(x, r))^{2} r^{-\beta-1} \mathrm{~d} r \mathrm{~d} x<+\infty$. We introduce the function $\varphi$ defined by

$$
\varphi(r)=\int_{\mathbb{R}^{d}} \mu(B(x, r))^{2} \mathrm{~d} x, r>0 .
$$

Using successively Fubini's Theorem, homogeneity and (11) we get

$$
\varphi(r)=\int_{\mathbb{R}^{d} \times \mathbb{R}^{d}}\left|B(z, r) \cap B\left(z^{\prime}, r\right)\right| \mu(\mathrm{d} z) \mu\left(\mathrm{d} z^{\prime}\right)=r^{d} \int_{\mathbb{R}^{d} \times \mathbb{R}^{d}} \gamma\left(\left|z-z^{\prime}\right| / r\right) \mu(\mathrm{d} z) \mu\left(\mathrm{d} z^{\prime}\right) .
$$

Therefore $\varphi(r) \leq \gamma(0)|\mu|\left(\mathbb{R}^{d}\right)^{2} r^{d}$. Moreover, since $\mu \in \widetilde{\mathcal{M}}_{\beta}$,

$$
\varphi(r)=r^{d} \int_{\mathbb{R}^{d} \times \mathbb{R}^{d}} \gamma_{\beta}\left(\left|z-z^{\prime}\right| / r\right) \mu(\mathrm{d} z) \mu\left(\mathrm{d} z^{\prime}\right) .
$$

and we can choose $\alpha$ such that $\int_{\mathbb{R}^{d} \times \mathbb{R}^{d}}\left|z-z^{\prime}\right|^{d-\alpha}|\mu|(\mathrm{d} z)|\mu|\left(\mathrm{d} z^{\prime}\right)<+\infty$ and (12) holds. Then

$$
\varphi(r) \leq C r^{\alpha} \int_{\mathbb{R}^{d} \times \mathbb{R}^{d}}\left|z-z^{\prime}\right|^{d-\alpha}|\mu|(\mathrm{d} z)|\mu|\left(\mathrm{d} z^{\prime}\right) .
$$

Finally, one can find $C>0$ such that

$$
\varphi(r) \leq C \min \left(r^{d}, r^{\alpha}\right),
$$

and

$$
\int_{0}^{+\infty} \varphi(r) r^{-\beta-1} \mathrm{~d} r=\int_{\mathbb{R}^{d} \times \mathbb{R}^{+}} \mu(B(x, r)) 2 r^{-\beta-1} \mathrm{~d} r \mathrm{~d} x<+\infty .
$$

2nd Step. We prove the equality stated in the Lemma, which can be formulated as

$$
C_{\beta} \int_{0}^{+\infty} \varphi(r) r^{-\beta-1} \mathrm{~d} r=c_{\beta} \int_{\mathbb{R}^{d} \times \mathbb{R}^{d}}\left|z-z^{\prime}\right|^{d-\beta} \mu(\mathrm{d} z) \mu\left(\mathrm{d} z^{\prime}\right),
$$


using the previous notations. For that purpose we want to replace $\varphi$ by (14) in the left hand side integral. Using the estimates (12) on $\left|\gamma_{\beta}\right|$, one can show that the integral defined by

$$
I_{\beta}(u):=\int_{\mathbb{R}^{+}} \gamma_{\beta}(u / r) r^{d-\beta-1} \mathrm{~d} r,
$$

is well defined for all $u \in \mathbb{R}_{+}$. Furthermore, $I_{\beta}$ is homogeneous of order $d-\beta$ so that

$$
\forall u>0, I_{\beta}(u)=I_{\beta}(1) u^{d-\beta} .
$$

This proves that

$$
\int_{0}^{+\infty} \varphi(r) r^{-\beta-1} \mathrm{~d} r=I_{\beta}(1) \int_{\mathbb{R}^{d} \times \mathbb{R}^{d}}\left|z-z^{\prime}\right|^{d-\beta} \mu(\mathrm{d} z) \mu\left(\mathrm{d} z^{\prime}\right),
$$

which completes the proof of the lemma with $c_{\beta}=C_{\beta} I_{\beta}(1)$.

Now let us state a second lemma which is the main tool to establish our scaling limit results.

Lemma 2.4. Let $F$ be a non-negative measure on $\mathbb{R}^{+}$satisfying $\mathbf{A}(\beta)$ for $\beta \neq d$.

(i) Assume that $g$ is a continuous function on $\mathbb{R}^{+}$such that for some $0<p<\beta<q$, there exists $C>0$ such that

$$
|g(r)| \leq C \min \left(r^{q}, r^{p}\right)
$$

Then

$$
\int_{\mathbb{R}^{+}} g(r) F_{\rho}(\mathrm{d} r) \sim C_{\beta} \rho^{\beta} \int_{\mathbb{R}^{+}} g(r) r^{-\beta-1} \mathrm{~d} r \text { as } \rho \rightarrow 0^{\beta-d} .
$$

(ii) Let $g_{\rho}$ be a family of continuous functions on $\mathbb{R}^{+}$. Assume that

$$
\lim _{\rho \rightarrow 0^{\beta-d}} \rho^{\beta} g_{\rho}(r)=0, \quad \text { and } \quad \rho^{\beta}\left|g_{\rho}(r)\right| \leq C \min \left(r^{p}, r^{q}\right),
$$

for some $0<p<\beta<q$ and $C>0$. Then

$$
\lim _{\rho \rightarrow 0^{\beta-d}} \int_{\mathbb{R}^{+}} g_{\rho}(r) F_{\rho}(\mathrm{d} r)=0 .
$$

Proof. (i) Let us assume for instance that $\beta<d$ (the proof of the case $\beta>d$ is similar and can be found in [13]). Let $\varepsilon>0$. Since $F$ satisfies $\mathbf{A}(\beta)$ there exists $\delta>0$ such that

$$
r<\delta \Rightarrow\left|f(r)-C_{\beta} r^{-\beta-1}\right| \leq \varepsilon r^{-\beta-1} .
$$

Let us remark that the assumptions on $g$ ensure that

$$
\int_{0}^{+\infty} g(r) r^{-\beta-1} \mathrm{~d} r<+\infty
$$

On the one hand, since $\int_{0}^{\delta \rho} g(r) F_{\rho}(\mathrm{d} r)=\int_{0}^{\delta \rho} g(r) f\left(\frac{r}{\rho}\right) \frac{\mathrm{d} r}{\rho}$, we get by (16)

$$
\left|\int_{0}^{\delta \rho} g(r) F_{\rho}(\mathrm{d} r)-C_{\beta} \rho^{\beta} \int_{0}^{\delta \rho} g(r) r^{-\beta-1} \mathrm{~d} r\right| \leq \varepsilon \rho^{\beta} \int_{\mathbb{R}^{+}} g(r) r^{-\beta-1} \mathrm{~d} r .
$$


On the other hand, for $\delta \rho>1$, since $|g(r)| \leq C r^{p}$,

$$
\begin{aligned}
\left|\int_{\delta \rho}^{\infty} g(r) F_{\rho}(\mathrm{d} r)-C_{\beta} \rho^{\beta} \int_{\delta \rho}^{\infty} g(r) r^{-\beta-1} \mathrm{~d} r\right| & \leq C_{1}(\delta) \rho^{p}+\frac{C_{\beta}}{\beta-p} \delta^{p-\beta} \rho^{p} \\
& \leq C(\delta) \rho^{p+1},
\end{aligned}
$$

where $C_{1}(\delta)=\int_{\delta}^{+\infty} r^{p} F(\mathrm{~d} r) \leq \delta^{p-d} \int_{\mathbb{R}^{+}} r^{d} F(\mathrm{~d} r)<\infty$. Since $p<\beta$, we obtain (i).

(ii) We follow the same lines as for (i) and can assume similarly that $\beta<d$. Since $F$ satisfies $\mathbf{A}(\beta)$ there exists $\delta>0$ such that

$$
r<\delta \Rightarrow|f(r)| \leq\left(C_{0}+1\right) r^{-\beta-1} .
$$

The assumptions on $g$ ensure that for all $\rho>0$,

$$
\int_{0}^{+\infty} \rho^{\beta}\left|g_{\rho}(r)\right| r^{-\beta-1} \mathrm{~d} r<+\infty
$$

Since $\int_{0}^{\delta \rho} g_{\rho}(r) F_{\rho}(\mathrm{d} r)=\int_{0}^{\delta \rho} g_{\rho}(r) f\left(\frac{r}{\rho}\right) \frac{\mathrm{d} r}{\rho}$, we get by $(17)$

$$
\left|\int_{0}^{\delta \rho} g_{\rho}(r) F_{\rho}(\mathrm{d} r)\right| \leq\left(C_{0}+1\right) \int_{0}^{\infty} \rho^{\beta}\left|g_{\rho}(r)\right| r^{-\beta-1} \mathrm{~d} r .
$$

Thus, by Lebesgue's Theorem

$$
\lim _{\rho \rightarrow+\infty} \int_{0}^{\delta \rho} g_{\rho}(r) F_{\rho}(\mathrm{d} r)=0 .
$$

Moreover, for $\delta \rho>1$, since $C_{1}(\delta)=\int_{\delta}^{+\infty} r^{p} F(\mathrm{~d} r)<+\infty$ and $\left|g_{\rho}(r)\right| \leq C \rho^{-\beta} r^{p}$,

$$
\begin{aligned}
\left|\int_{\delta \rho}^{\infty} g_{\rho}(r) F_{\rho}(\mathrm{d} r)\right| & \leq C \rho^{-\beta} \int_{\delta \rho}^{\infty} r^{p} F_{\rho}(\mathrm{d} r) \\
& \leq C C_{1}(\delta) \rho^{-(\beta-p)} .
\end{aligned}
$$

We conclude the proof using (18) and (19), since $p<\beta$.

We start now with the proof of Theorem 2.1. Let us denote

$$
n(\rho):=\sqrt{\lambda(\rho) \rho^{\beta}}
$$

and define the function $\varphi_{\rho}$ on $\mathbb{R}^{+}$by

$$
\varphi_{\rho}(r)=\int_{\mathbb{R}^{d}} \Psi\left(\frac{\mu(B(x, r))}{n(\rho)}\right) \mathrm{d} x,
$$

where

$$
\Psi(v)=e^{i v}-1-i v .
$$

The characteristic function of the normalized field $\left(X_{\rho}()-.\mathbb{E}\left(X_{\rho}().\right)\right) / n(\rho)$ is given by

$$
\mathbb{E}\left(\exp \left(i \frac{X_{\rho}(\mu)-\mathbb{E}\left(X_{\rho}(\mu)\right)}{n(\rho)}\right)\right)=\exp \left(\int_{\mathbb{R}^{+}} \lambda(\rho) \varphi_{\rho}(r) F_{\rho}(\mathrm{d} r)\right) .
$$


By assumption, $n(\rho)$ tends to $+\infty$ as $\rho \rightarrow 0^{\beta-d}$ so that $\Psi\left(\frac{\mu(B(x, r))}{n(\rho)}\right)$ behaves like $-\frac{1}{2}\left(\frac{\mu(B(x, r))}{n(\rho)}\right)^{2}$. Therefore, we write

$$
\int_{\mathbb{R}^{+}} \lambda(\rho) \varphi_{\rho}(r) F_{\rho}(\mathrm{d} r)=-\frac{1}{2} \int_{\mathbb{R}^{+}} \varphi(r) \lambda(\rho) n(\rho)^{-2} F_{\rho}(\mathrm{d} r)+\int_{\mathbb{R}^{+}} \Delta_{\rho}(r) F_{\rho}(\mathrm{d} r)
$$

where the function $\varphi$ is introduced in (13) and

$$
\begin{aligned}
\Delta_{\rho}(r) & =\lambda(\rho) \varphi_{\rho}(r)+\frac{1}{2} \lambda(\rho) n(\rho)^{-2} \varphi(r) \\
& =\lambda(\rho) \int_{\mathbb{R}^{d}}\left(\Psi\left(\frac{\mu(B(x, r))}{n(\rho)}\right)+\frac{1}{2}\left(\frac{\mu(B(x, r)}{n(\rho)}\right)^{2}\right) \mathrm{d} x .
\end{aligned}
$$

Since $\mu \in \widetilde{\mathcal{M}}_{\beta}$, the function $\varphi$ satisfies (15) and thus the assumptions of Lemma 2.4(i). Thus, the first term of the right hand side of (21) converges to $C_{\beta} \int_{\mathbb{R}^{+}} \varphi(r) r^{-\beta-1} \mathrm{~d} r$, and using Lemma 2.2 we obtain

$$
\lim _{\rho \rightarrow 0^{\beta-d}} \int_{\mathbb{R}^{+}} \varphi(r) \lambda(\rho) n(\rho)^{-2} F_{\rho}(\mathrm{d} r)=c_{\beta} \int_{\mathbb{R}^{d} \times \mathbb{R}^{d}}\left|z-z^{\prime}\right|^{d-\beta} \mu(\mathrm{d} z) \mu\left(\mathrm{d} z^{\prime}\right) .
$$

For the second term, let us verify that $\Delta_{\rho}$ given by (22) satisfies the assumptions of Lemma 2.4(ii). First let us remark that the function $\Delta_{\rho}$ is continuous on $\mathbb{R}^{+}$since $\mu \in \mathcal{M}$. Because $\left|\Psi(v)-\left(-\frac{v^{2}}{2}\right)\right| \leq \frac{|v|^{3}}{6}$ and

$$
\int_{\mathbb{R}^{d}} \mu(B(x, r))^{3} \mathrm{~d} x \leq\|\mu\|_{1}^{2} \int_{\mathbb{R}^{d}}|\mu(B(x, r))| \mathrm{d} x \leq C_{d}\|\mu\|_{1}^{3} r^{d},
$$

we also check that

$$
\left|\lambda(\rho)^{-1} n(\rho)^{2} \Delta_{\rho}(r)\right| \leq \frac{C_{d}\|\mu\|_{1}^{3}}{6} n(\rho)^{-1} r^{d} .
$$

Finally, since $|\Psi(v)| \leq \frac{|v|^{2}}{2}$, there exists $\alpha$ with $(\alpha-\beta)(\beta-d)>0$ such that by (15)

$$
\left|\lambda(\rho)^{-1} n(\rho)^{2} \Delta_{\rho}(r)\right| \leq C r^{\alpha} .
$$

Therefore, $\int_{\mathbb{R}^{+}} \Delta_{\rho}(r) F_{\rho}(\mathrm{d} r)$ tends to 0 according to Lemma 2.4(ii), and so

$$
\lim _{\rho \rightarrow 0^{\beta-d}} \mathbb{E}\left(\exp \left(i \frac{X_{\rho}(\mu)-\mathbb{E}\left(X_{\rho}(\mu)\right)}{n(\rho)}\right)\right)=\exp \left(-\frac{1}{2} c_{\beta} \int_{\mathbb{R}^{d} \times \mathbb{R}^{d}}\left|z-z^{\prime}\right|^{d-\beta} \mu(\mathrm{d} z) \mu\left(\mathrm{d} z^{\prime}\right)\right) \text {. }
$$

Hence $\left(X_{\rho}(\mu)-\mathbb{E}\left(X_{\rho}(\mu)\right)\right) / n(\rho)$ converges in distribution to the centered Gaussian random variable $W(\mu)$ whose variance is equal to

$$
\mathbb{E}\left(W(\mu)^{2}\right)=c_{\beta} \int_{\mathbb{R}^{d} \times \mathbb{R}^{d}}\left|z-z^{\prime}\right|^{d-\beta} \mu(\mathrm{d} z) \mu\left(\mathrm{d} z^{\prime}\right) .
$$

By linearity, the covariance of $W$ satisfies (10).

With similar arguments we can state an other scaling result leading to a non-Gaussian limit. 
2.3. Poisson limit regime. In this section we keep the notations introduced in section 2.2 for the Gaussian limit regime.

Theorem 2.5. Let $d-1<\beta<2 d$ such that $\beta \neq d$. Let $F$ be a non-negative measure on $\mathbb{R}^{+}$satisfying $\mathbf{A}(\beta)$. For all positive functions $\lambda$ such that $\lambda(\rho) \rho^{\beta} \underset{\rho \rightarrow 0^{\beta-d}}{\longrightarrow} a^{d-\beta}$, for some $a>0$, we have in the sense of finite-dimensional distributions of random functionals the scaling limit

$$
X_{\rho}(\mu)-\mathbb{E}\left(X_{\rho}(\mu)\right) \stackrel{f d d}{\rightarrow} J_{\beta}\left(\mu_{a}\right),
$$

for all $\mu \in \widetilde{\mathcal{M}}_{\beta}$. Here $J_{\beta}$ is the centered random linear functional on $\widetilde{\mathcal{M}}_{\beta}$ defined as

$$
J_{\beta}(\mu)=\int_{\mathbb{R}^{d} \times \mathbb{R}^{+}} \mu(B(x, r)) \widetilde{N_{\beta}}(\mathrm{d} x, \mathrm{~d} r),
$$

where $\widetilde{N_{\beta}}$ is a compensated Poisson random measure with intensity $C_{\beta} \mathrm{d} x r^{-\beta-1} \mathrm{~d} r$, and $\mu_{a}$ is defined by $\mu_{a}(A)=\mu\left(a^{-1} A\right)$.

Proof. The stochastic integral $\int k(x, r) \tilde{N}(\mathrm{~d} x, \mathrm{~d} r)$ of a measurable function $k: \mathbb{R}^{d} \times \mathbb{R}^{+} \rightarrow$ $\mathbb{R}$ with respect to a compensated Poisson measure $\tilde{N}$ of intensity $n$, exists $\mathbb{P}$-a.s. if and only if

$$
\int_{\mathbb{R}^{d} \times \mathbb{R}^{+}} \min \left(|k(x, r)|, k(x, r)^{2}\right) n(\mathrm{~d} x, \mathrm{~d} r)<\infty
$$

(see [15] for instance). $(13)$,

By Lemma 2.2, for all $\mu \in \widetilde{\mathcal{M}}_{\beta}$ and using once again the function $\varphi$ introduced in

$$
\int_{\mathbb{R}^{d}} \int_{\mathbb{R}^{+}} \mu(B(x, r))^{2} r^{-\beta-1} \mathrm{~d} r \mathrm{~d} x=\int_{\mathbb{R}^{+}} \varphi(r) r^{d-\beta-1} \mathrm{~d} r<+\infty .
$$

Hence, in view of (23) with $n(\mathrm{~d} x, \mathrm{~d} r)=C_{\beta} \mathrm{d} x r^{-\beta-1} \mathrm{~d} r$ and $k(x, r)=\mu(B(x, r))$, the random field $J_{\beta}$ is well defined on $\widetilde{\mathcal{M}}_{\beta}$, with characteristic function

$$
\mathbb{E}\left(\exp \left(i J_{\beta}(\mu)\right)\right)=\exp \left(\int_{\mathbb{R}^{+} \times \mathbb{R}^{d}} \Psi(\mu(B(x, r))) C_{\beta} \mathrm{d} x r^{-\beta-1} \mathrm{~d} r\right),
$$

where $\Psi$ is given by $(20)$.

On the other hand, the characteristic function for the centered Poisson random balls model equals

$$
\mathbb{E}\left(\exp \left(i\left(X_{\rho}(\mu)-\mathbb{E}\left(X_{\rho}(\mu)\right)\right)\right)=\exp \left(\int_{\mathbb{R}^{+} \times \mathbb{R}^{d}} \Psi(\mu(B(x, r))) \mathrm{d} x \lambda(\rho) F_{\rho}(\mathrm{d} r)\right) .\right.
$$

Define for $r>0$,

$$
\widetilde{\varphi}(r)=\int_{\mathbb{R}^{d}} \Psi(\mu(B(x, r))) \mathrm{d} x .
$$

For $\mu \in \widetilde{\mathcal{M}}_{\beta}$, using $|\Psi(v)| \leq|v|^{2} / 2$ and (15), there exists $C>0$ such that

$$
|\widetilde{\varphi}(r)| \leq C \min \left(r^{d}, r^{\alpha}\right),
$$


for some $\alpha$ with $(\alpha-\beta)(\beta-d)>0$. Thus, by Lemma 2.4 (i),

$$
\int_{\mathbb{R}^{+}} \lambda(\rho) \widetilde{\varphi}(r) F_{\rho}(\mathrm{d} r) \underset{\rho \rightarrow 0^{\beta-d}}{\sim} C_{\beta} a^{d-\beta} \int_{0}^{\infty} \widetilde{\varphi}(r) r^{-\beta-1} \mathrm{~d} r
$$

and hence

$$
\lim _{\rho \rightarrow 0^{\beta-d}} \mathbb{E}\left(\exp \left(i\left(X_{\rho}(\mu)-\mathbb{E}\left(X_{\rho}(\mu)\right)\right)\right)=\exp \left(C_{\beta} a^{d-\beta} \int_{\mathbb{R}^{+}} \widetilde{\varphi}(r) r^{-\beta-1} \mathrm{~d} r\right) .\right.
$$

Finally, it is sufficient to remark that

$$
a^{d-\beta} \int_{\mathbb{R}^{+}} \widetilde{\varphi}(r) r^{-\beta-1} \mathrm{~d} r=a^{d} \int_{\mathbb{R}^{+}} \widetilde{\varphi}\left(a^{-1} r\right) r^{-\beta-1} \mathrm{~d} r
$$

with

$$
a^{d} \widetilde{\varphi}\left(a^{-1} r\right)=a^{d} \int_{\mathbb{R}^{d}} \Psi\left(\mu\left(B\left(x, a^{-1} r\right)\right)\right) \mathrm{d} x=\int_{\mathbb{R}^{d}} \Psi\left(\mu_{a}(B(x, r))\right) \mathrm{d} x,
$$

to obtain

$$
\lim _{\rho \rightarrow 0^{\beta-d}} \mathbb{E}\left(\exp \left(i\left(X_{\rho}(\mu)-\mathbb{E}\left(X_{\rho}(\mu)\right)\right)\right)\right)=\mathbb{E}\left(\exp \left(i J_{\beta}\left(\mu_{a}\right)\right)\right)
$$

Lemma 2.2 and (10) yield the following remark.

Remark 2.6. The covariance function of $J_{\beta}$ is given for all $\mu, \nu \in \widetilde{\mathcal{M}}_{\beta}$ by

$$
\begin{aligned}
\operatorname{Cov}\left(J_{\beta}(\mu), J_{\beta}(\nu)\right) & =\int_{\mathbb{R}^{d} \times \mathbb{R}^{+}} \mu(B(x, r)) \nu(B(x, r)) C_{\beta} \mathrm{d} x r^{-\beta-1} \mathrm{~d} r \\
& =c_{\beta} \int_{\mathbb{R}^{d} \times \mathbb{R}^{d}}\left|z-z^{\prime}\right|^{d-\beta} \mu(\mathrm{d} z) \nu\left(\mathrm{d} z^{\prime}\right),
\end{aligned}
$$

and so $J_{\beta}$ and $W_{\beta}$ have the same covariance function on $\widetilde{\mathcal{M}}_{\beta}$.

A stable limit regime should also be obtained as in [13], under the assumption that $\lambda(\rho) \rho^{\beta} \rightarrow 0$ as $\rho \rightarrow 0^{\beta-d}$. The limit is expected to be an independently scattered $\beta / d$-stable random measure on $\mathbb{R}^{d}$ with Lebesgue control measure and unit skewness.

2.4. Properties of the limiting random generalized fields. We complete this section by discussing some of the main properties of the fields we obtain as scaling limits. Due to the stationarity of the random balls field on the one hand and to the performed scaling on the other hand, the limits inherit a stationarity property. The Gaussian limits exhibit a self-similarity property as well.

Following the same ideas as in [7] or [16] we define a notion of stationarity which characterizes the translation invariance of a random linear functional over a subset of signed measures. We say as usual that a subspace $\mathcal{S} \subset \mathcal{M}$ is closed for translations if, for any $\mu \in \mathcal{S}$ and any $s \in \mathbb{R}^{d}$, we have $\tau_{s} \mu \in \mathcal{S}$, where $\tau_{s} \mu$ is defined by $\tau_{s} \mu(A)=\mu(A-s)$, for any Borel set $A$. To provide a more general framework for stationary random fields we introduce the following subspaces of measures with vanishing moments. For any $n \in \mathbb{N} \backslash\{0\}$, denote by $\mathcal{M}_{n}$ the subspace of measures $\mu \in \mathcal{M}$, which satisfy

$$
\int_{\mathbb{R}^{d}} z^{j} \mu(\mathrm{d} z)=\int_{\mathbb{R}^{d}} z_{1}^{j_{1}} \ldots z_{d}^{j_{d}} \mu(\mathrm{d} z)=0
$$


for all $j=\left(j_{1}, \ldots, j_{d}\right) \in \mathbb{N}^{d}$ with $0 \leq j_{1}+\ldots+j_{d}<n$ (see [16] where similar spaces of measures are introduced). Here, the class $\mathcal{M}_{1}$ was already used for the setting of Theorem 2.1. For convenience, we also put $\mathcal{M}_{0}=\mathcal{M}$. A simple but tedious computation shows that

$$
\mathcal{M}_{n}=\left\{\mu \in \mathcal{M}: \int_{\mathbb{R}^{d} \times \mathbb{R}^{d}}\left|z-z^{\prime}\right|^{2 k} \mu(\mathrm{d} z) \mu\left(\mathrm{d} z^{\prime}\right)=0 \text { for all integers } 0 \leq k<n\right\} .
$$

In particular, the subspaces $\mathcal{M}_{n}$, defined by (25), are closed for translations for any $n \in \mathbb{N}$.

Definition 2.7. Let $n \in \mathbb{N}$. Let $X$ be a random field defined on a subspace $\mathcal{S} \subset \mathcal{M}_{n}$ closed for translations. The field $X$ is translation invariant if

$$
\forall \mu \in \mathcal{S}, \forall s \in \mathbb{R}^{d}, X\left(\tau_{s} \mu\right) \stackrel{\text { fdd }}{=} X(\mu) .
$$

More precisely, one says that $X$ is stationary when $n=0$ and has stationary $n$th increments when $n>0$.

It follows that if $X$ has stationary $n$th increments on a subspace $\mathcal{S} \subset \mathcal{M}_{n}$, then its restriction on $\mathcal{S} \cap \mathcal{M}_{n+1} \subset \mathcal{M}_{n+1}$ has stationary $(n+1)$ th increments.

By the translation invariance of the Lebesgue measure, for any $\rho>0$ the random field $X_{\rho}$ defined by (6) is stationary on $\mathcal{M}$. The fields $W_{\beta}$ and $J_{\beta}$ obtained as limit fields on $\widetilde{\mathcal{M}}_{\beta}$ in Theorem 2.1 and Theorem 2.5 are not defined on the full space $\mathcal{M}$. But $\widetilde{\mathcal{M}}_{\beta}$ is closed for translations. Therefore, when considering the limiting random fields on $\widetilde{\mathcal{M}}_{\beta}$, one has the following property.

Proposition 2.8. Let $d-1<\beta<2 d$ with $\beta \neq d$. Then $W_{\beta}$ as well as $J_{\beta}$ are translation invariant on $\widetilde{\mathcal{M}}_{\beta}$.

In other words, from (9), $W_{\beta}$ and $J_{\beta}$ defined on $\widetilde{\mathcal{M}}_{\beta}$ are both stationary if $d<\beta<2 d$ and they have stationary first increments if $d-1<\beta<d$.

We turn now to the self-similarity property. Denote by $\mu_{a}$ the dilated measure defined by $\mu_{a}(A)=\mu\left(a^{-1} A\right)$ for any Borel set $A$. A subspace $\mathcal{S} \subset \mathcal{M}$ is said to be closed for dilations if, for any $\mu \in \mathcal{S}$ and any $a>0$, we have $\mu_{a} \in \mathcal{S}$. The following definition extends the standard definition of self-similarity for pointwise defined random fields.

Definition 2.9. Let $H \in \mathbb{R}$. A random field $X$, defined on a subspace $\mathcal{S}$ of $\mathcal{M}$ which is closed for dilations, is said to be self-similar with index $H$ if

$$
\forall \mu \in \mathcal{S}, \forall a>0, X\left(\mu_{a}\right) \stackrel{f d d}{=} a^{H} X(\mu) .
$$

Once noticed that $\widetilde{\mathcal{M}}_{\beta}$ is closed for dilations, and by observing the consequence of dilation on the covariance of $W_{\beta}$, the following property is straightforward.

Proposition 2.10. The field $W_{\beta}$, defined on $\widetilde{\mathcal{M}}_{\beta}$, is self-similar with index $H=\frac{d-\beta}{2}$ that runs over $(-d / 2,1 / 2) \backslash\{0\}$.

In contrast to the Gaussian field $W_{\beta}$, the Poisson limit field $J_{\beta}$ is not self-similar. 


\section{SELF-SIMILAR RANDOM FIELDS OF ARBITRARY ORDER}

3.1. Dobrushin's characterization. Dobrushin [7] gives a complete description of Gaussian translation invariant self-similar generalized random fields on $\mathbb{R}^{d}$. For this purpose he considers continuous random linear functionals of $\mathcal{S}\left(\mathbb{R}^{d}\right)^{\prime}$, where $\mathcal{S}\left(\mathbb{R}^{d}\right)^{\prime}$ is the topological dual of the Schwartz space $\mathcal{S}\left(\mathbb{R}^{d}\right)$ of all infinitely differentiable rapidly decreasing functions on $\mathbb{R}^{d}$ (see e.g. [8]). As usual $\mathcal{S}\left(\mathbb{R}^{d}\right)$ is equipped with the topology that corresponds to the following notion of convergence: $\varphi_{n} \rightarrow \varphi$ if and only if for all $N \in \mathbb{N}$ and $j \in \mathbb{N}^{d}$

$$
\sup _{z \in \mathbb{R}^{d}}(1+|z|)^{N}\left|D^{j}\left(\varphi_{n}-\varphi\right)(z)\right| \rightarrow 0
$$

where $D^{j} \varphi(z)=\frac{\partial^{j_{1}} \ldots \partial^{j_{d}}}{\partial z_{1}^{j_{1}} \ldots \partial z_{d}^{j_{d}}} \varphi(z)$ denotes the partial derivative of order $j=\left(j_{1}, \ldots, j_{d}\right)$. Then, a linear functional $X: \mathcal{S}\left(\mathbb{R}^{d}\right) \rightarrow L^{2}(\Omega, \mathcal{A}, \mathbb{P})$ is continuous if and only if $\varphi_{n} \rightarrow 0$ in $\mathcal{S}\left(\mathbb{R}^{d}\right)$ implies that

$$
\mathbb{E}\left(X\left(\varphi_{n}\right)^{2}\right) \rightarrow 0
$$

To each function $\varphi \in \mathcal{S}\left(\mathbb{R}^{d}\right) \subset L^{1}\left(\mathbb{R}^{d}\right)$ one can uniquely associate a signed measure $\widetilde{\varphi} \in \mathcal{M}$ defined by $\widetilde{\varphi}(\mathrm{d} z)=\varphi(z) \mathrm{d} z$. For the sake of notational simplicity we identify any function $\varphi \in L^{1}\left(\mathbb{R}^{d}\right)$ with its image $\widetilde{\varphi}$ in $\mathcal{M}$ so that $L^{1}\left(\mathbb{R}^{d}\right) \subset \mathcal{M}$. Therefore any random linear functional on $\mathcal{M}$, when restricted to $\mathcal{S}\left(\mathbb{R}^{d}\right)$, can be viewed as a linear functional on $\mathcal{S}\left(\mathbb{R}^{d}\right)$.

Proposition 3.1. Let $\rho>0$. The random field $X_{\rho}$ induces a continuous random linear functional on $\mathcal{S}\left(\mathbb{R}^{d}\right)$.

Proof. ¿From (8), the random field $X_{\rho}$ is a continuous random linear functional on $\mathcal{M}$. Then, to prove the continuity of $X_{\rho}$ on $\mathcal{S}\left(\mathbb{R}^{d}\right)$ it is sufficient, using Lebesgue's theorem, to notice that the previous identification implies that if $\mu_{n}=\widetilde{\varphi_{n}} \rightarrow 0$ in $\mathcal{S}\left(\mathbb{R}^{d}\right)$ then $\mu_{n} \rightarrow 0$ in $\mathcal{M}$.

Now, put

$$
\mathcal{S}_{n}\left(\mathbb{R}^{d}\right)=\mathcal{S}\left(\mathbb{R}^{d}\right) \cap \mathcal{M}_{n}, \quad n \geq 0 .
$$

In particular, $\mathcal{S}_{0}\left(\mathbb{R}^{d}\right)=\mathcal{S}\left(\mathbb{R}^{d}\right)$. We obtain the continuity properties of $W_{\beta}$ and $J_{\beta}$ by observing that $\mathcal{S}\left(\mathbb{R}^{d}\right) \cap \widetilde{\mathcal{M}}_{\beta}=\mathcal{S}\left(\mathbb{R}^{d}\right)$ when $d<\beta<2 d$, while $\mathcal{S}\left(\mathbb{R}^{d}\right) \cap \widetilde{\mathcal{M}}_{\beta}=$ $\mathcal{S}\left(\mathbb{R}^{d}\right) \cap \mathcal{M}_{1}=\mathcal{S}_{1}\left(\mathbb{R}^{d}\right)$ for $d-1<\beta<d$.

Proposition 3.2. Let $d-1<\beta<2 d$ with $\beta \neq d$. The random fields $W_{\beta}$ and $J_{\beta}$ induce continuous random linear functionals on $\mathcal{S}_{n}\left(\mathbb{R}^{d}\right)$ for any $n \geq 1$ if $d-1<\beta<d$, and any $n \geq 0$ if $d<\beta<2 d$.

Proof. Note that by (10) and Remark 2.6, for any $\mu \in \widetilde{\mathcal{M}}_{\beta}$,

$$
\mathbb{E}\left(W_{\beta}(\mu)^{2}\right)=\mathbb{E}\left(J_{\beta}(\mu)^{2}\right) \leq\left|c_{\beta}\right| \int_{\mathbb{R}^{d} \times \mathbb{R}^{d}}\left|z-z^{\prime}\right|^{d-\beta}|\mu|(\mathrm{d} z)|\mu|\left(\mathrm{d} z^{\prime}\right) .
$$

A straightforward use of Lebesgue's theorem concludes the proof.

Then, restricted to $\mathcal{S}_{n}\left(\mathbb{R}^{d}\right)$ the Gaussian field $W_{\beta}$ is a translation invariant self-similar generalized field. We refer to [17] for a synthesis using orthonormal basis of $L^{2}\left(\mathbb{R}^{d}\right)$ 
in the case $d<\beta<2 d$ and to [6] for other examples of self-similar generalized fields obtained by random wavelet expansions in the general case. In [7] Dobrushin focuses on the spectral representation of such Gaussian fields. Since the law of a centered Gaussian field is characterized by its covariance function, let us introduce a second order selfsimilarity property. For $H \in \mathbb{R}$ we say that $X$, a random linear functional on $\mathcal{S}_{n}\left(\mathbb{R}^{d}\right)$ is a second order self-similar field of order $H$ if, for all $a>0, \varphi, \psi \in \mathcal{S}_{n}\left(\mathbb{R}^{d}\right)$,

$$
\operatorname{Cov}\left(X\left(\varphi_{a}\right), X\left(\psi_{a}\right)\right)=a^{2 H} \operatorname{Cov}(X(\varphi), X(\psi)) \text {, where } \varphi_{a}(x)=a^{-d} \varphi\left(a^{-1} x\right) .
$$

We denote by $\widehat{\mu}(\xi)=\int_{\mathbb{R}^{d}} e^{-i z \cdot \xi} \mu(\mathrm{d} z)$ the Fourier transform of any $\mu \in \mathcal{M}$. Then Theorem 3.2 of [7] can be reformulated as follows.

Theorem 3.3. Let $n \geq 0$ and $X$ be a continuous random linear functional on $\mathcal{S}_{n}\left(\mathbb{R}^{d}\right)$. Then $X$ is translation invariant and second order self-similar field of order $H \in \mathbb{R}$ if and only if for all $\varphi, \psi \in \mathcal{S}_{n}\left(\mathbb{R}^{d}\right)$,

$$
\operatorname{Cov}(X(\varphi), X(\psi))=\int_{S^{d-1}} \int_{\mathbb{R}^{+}} \widehat{\varphi}(r \theta) \overline{\widehat{\psi}}(r \theta) r^{-2 H-1} \mathrm{~d} r \mathrm{~d} \sigma(\theta)+\sum_{|j|=|k|=n} A_{j, k} \alpha_{j}(\varphi) \overline{\alpha_{k}(\psi)},
$$

where $\sigma$ is a finite positive measure on the unit sphere $S^{d-1}, \alpha_{j}(\varphi)=\int_{\mathbb{R}^{d}} \varphi(x) x^{j} \mathrm{~d} x=$ $i^{|j|} D^{j} \widehat{\varphi}(0)$, for $j \in \mathbb{N}^{d}$ with $|j|=n$, and $A=\left(A_{j, k}\right)_{|j|=|k|=n}$ is a symmetric positive definite real matrix. Moreover, if $H<n$ then $A=0$; if $H=n$ then $\sigma=0$; and if $H>n$ then $A=0$ and $\sigma=0$.

We make the further comment that generalized random fields defined on $\mathcal{S}_{n}\left(\mathbb{R}^{d}\right)$ for some $n>0$ roughly correspond to suitable derivatives of random fields defined on $\mathcal{S}\left(\mathbb{R}^{d}\right)$. More precisely, since the Schwartz class is closed under differentiation, if $X$ is a continuous random linear functional on $\mathcal{S}\left(\mathbb{R}^{d}\right)$ one can define for any $j \in \mathbb{N}^{d}$ the partial derivative of $X$ of order $j$ as the continuous random linear functional defined by

$$
\forall \varphi \in \mathcal{S}\left(\mathbb{R}^{d}\right), \quad D^{j} X(\varphi)=(-1)^{|j|} X\left(D^{j} \varphi\right) .
$$

Moreover, Lemma 1.2.1 p.23 of [3] states the following property.

Proposition 3.4. For any $n \in \mathbb{N}, \mathcal{S}_{n}\left(\mathbb{R}^{d}\right)=\operatorname{Span}\left\{D^{j} \varphi: \varphi \in \mathcal{S}\left(\mathbb{R}^{d}\right), j \in \mathbb{N}^{d},|j|=n\right\}$.

Therefore, the knowledge of a generalized random field $X$ on $\mathcal{S}_{n}\left(\mathbb{R}^{d}\right)$ is equivalent to the knowledge of all its partial derivatives $D^{j} X$ of order $j$ with $|j|=n$. Furthermore, $X$ has stationary $n$th increments if and only if its partial derivatives $D^{j} X$ of order $j$ with $|j|=n$ are stationary.

Note that $W_{\beta}$ and $J_{\beta}$ share the same covariance function by Remark 2.6 so that they are both second order self-similar fields of order $\frac{d-\beta}{2}$. Therefore, by Proposition 3.2 and Theorem 3.3 they have to be considered on $\mathcal{S}_{n}\left(\mathbb{R}^{d}\right)$ for $n \geq((d-\beta) / 2)_{+}$. Moreover, due to the isotropy of balls and the rotation invariance of Lebesgue's measure it is straightforward to conclude that $W_{\beta}$ and $J_{\beta}$ are isotropic random fields. Thus, the measure $\sigma$ obtained from Theorem 3.3 is invariant under rotation and hence, up to a constant, Lebesgue's measure on the sphere. We obtain the following result, which is of Plancherel's type and gives the covariance function of $W_{\beta}$ and $J_{\beta}$ in spectral form. 
Proposition 3.5. Fix $d-1<\beta<2 d$ with $\beta \neq d$. There exists $k_{\beta}>0$ such that, if $d<\beta<2 d$ then for any $\varphi, \psi \in \mathcal{S}\left(\mathbb{R}^{d}\right)$ and if $d-1<\beta<d$ for any $\varphi, \psi \in \mathcal{S}_{1}\left(\mathbb{R}^{d}\right)$, we have

$$
\begin{aligned}
& \operatorname{Cov}\left(W_{\beta}(\varphi), W_{\beta}(\psi)\right)=\operatorname{Cov}\left(J_{\beta}(\varphi), J_{\beta}(\psi)\right) \\
& \quad=c_{\beta} \int_{\mathbb{R}^{d} \times \mathbb{R}^{d}}\left|z-z^{\prime}\right|^{d-\beta} \varphi(z) \psi\left(z^{\prime}\right) \mathrm{d} z \mathrm{~d} z^{\prime}=k_{\beta} \int_{\mathbb{R}^{d}} \widehat{\varphi}(\xi) \overline{\widehat{\psi}}(\xi)|\xi|^{\beta-2 d} \mathrm{~d} \xi
\end{aligned}
$$

3.2. Higher order stationary random fields as scaling limits. To exploit Dobrushin's characterization theorem further, we consider next a general class of Gaussian random fields. Let $H \in \mathbb{R}$ with $H \notin \mathbb{N}$ be an arbitrary self-similarity index and write

$$
\lceil H\rceil_{+}= \begin{cases}{[H]+1,} & H>0 \\ 0, & H<0,\end{cases}
$$

where $[H]$ is the integer part of $H$. Let $B_{H}$ denote a continuous random field defined on $\mathcal{S}_{\lceil H\rceil_{+}}$, which is centered, Gaussian and isotropic, and such that for some constant $k_{H}>0$ the covariance functional is given by

$$
\operatorname{Cov}\left(B_{H}(\varphi), B_{H}(\psi)\right)=k_{H} \int_{\mathbb{R}^{d}} \widehat{\varphi}(\xi) \overline{\widehat{\psi}}(\xi)|\xi|^{-2 H-d} \mathrm{~d} \xi, \quad \varphi, \psi \in \mathcal{S}_{\lceil H\rceil_{+}}\left(\mathbb{R}^{d}\right) .
$$

In the special case $H>0$, for which $\lceil H\rceil_{+}=\lceil H\rceil$, we note that $\lceil H\rceil-1<H<\lceil H\rceil$ and that the random field $B_{H}$ has stationary increments of order $\lceil H\rceil$. It is straightforward to show that for all $j \in \mathbb{N}^{d}$ with $|j|=\lceil H\rceil$, the corresponding differentiated field $D^{j} B_{H}$ is stationary and admits a spectral density which behaves near the origin as $|\xi|^{-2 H-d+2\lceil H\rceil}$. In this situation, following the usual interpretation of the spectral density close to the origin, we say that $B_{H}$ provides long range dependence as soon as $-2 H+2\lceil H\rceil$ is less than 1 , which is equivalent to $\lceil H\rceil-1 / 2<H$. In what follows we will see that for $H$ such that $\lceil H\rceil-1 / 2<H<\lceil H\rceil$, the field $B_{H}$ may be explicitly constructed as the scaling limit of a random germ-grain model where grains have a heavy-tailed radius distribution at infinity. The overlap of these large grains yields a strong spatial dependence. In the opposite case, $\lceil H\rceil-1<H<\lceil H\rceil-1 / 2$, the field $B_{H}$ may be explicitly constructed as the scaling limit of a random germ-grain model where the radius of grains accumulates at zero. The interaction of the small grains yields a negative type correlation.

We return to the case of real, non-integer $H$. In order to link the Dobrushin fields $B_{H}$ and the limit fields $W_{\beta}$ we obtained in previous section, we will use fractional integration and differentiation. In [17] a similar procedure is used to synthesize Gaussian self-similar random fields with $H \in(-d / 2,0)$. To introduce the method we consider for $\varphi \in \mathcal{S}\left(\mathbb{R}^{d}\right)$ the usual Laplacian operator

$$
\Delta \varphi=\sum_{j=1}^{d} \frac{\partial^{2} \varphi}{\partial z_{j}^{2}}
$$

and recall that for any $\xi \in \mathbb{R}^{d}$,

$$
\widehat{\Delta \varphi}(\xi)=-|\xi|^{2} \widehat{\varphi}(\xi)
$$


Next, for any $m \in \mathbb{Z}$, we may define formally the operator $(-\Delta)^{-\frac{m}{2}}$ by the relation

$$
\left(-\widehat{\Delta)^{-\frac{m}{2}}} \varphi(\xi)=|\xi|^{-m} \widehat{\varphi}(\xi), \quad \xi \in \mathbb{R}^{d} .\right.
$$

In order to give a precise meaning to this operator, we introduce the intersection space

$$
\mathcal{S}_{\infty}\left(\mathbb{R}^{d}\right)=\bigcap_{n \geq 0} \mathcal{S}_{n}\left(\mathbb{R}^{d}\right)
$$

Proposition 3.6. For any $m \in \mathbb{Z}$, the operator $(-\Delta)^{-\frac{m}{2}}: \mathcal{S}_{\infty}\left(\mathbb{R}^{d}\right) \longrightarrow \mathcal{S}_{\infty}\left(\mathbb{R}^{d}\right)$ is continuous.

Proof. For any $n \geq 1$,

$$
\mathcal{S}_{n}\left(\mathbb{R}^{d}\right)=\left\{\varphi \in \mathcal{S}\left(\mathbb{R}^{d}\right) ; D^{j} \widehat{\varphi}(0)=0,|j|<n\right\} .
$$

Thus, $\mathcal{S}_{\infty}\left(\mathbb{R}^{d}\right) \neq \emptyset$ since this space contains any function $\varphi \in \mathcal{S}\left(\mathbb{R}^{d}\right)$ such that $\widehat{\varphi}$ vanishes in a neighborhood of 0 . It is therefore clear that $\widehat{(-\Delta)^{-\frac{m}{2}}}: \mathcal{F}\left(\mathcal{S}_{\infty}\left(\mathbb{R}^{d}\right)\right) \longrightarrow \mathcal{F}\left(\mathcal{S}_{\infty}\left(\mathbb{R}^{d}\right)\right)$ is continuous with $\mathcal{F}\left(\mathcal{S}_{\infty}\left(\mathbb{R}^{d}\right)\right)=\left\{\widehat{\varphi} ; \varphi \in \mathcal{S}_{\infty}\left(\mathbb{R}^{d}\right)\right\}$. The proof is completed by using the continuity on $\mathcal{F}\left(\mathcal{S}_{\infty}\left(\mathbb{R}^{d}\right)\right) \subset \mathcal{S}\left(\mathbb{R}^{d}\right)$ of the Fourier transform inverse.

Theorem 3.7. Let $H \in \mathbb{R}$ with $H \notin \frac{1}{2} \mathbb{Z}$ for $d=1$ and $H \notin \mathbb{Z}$ for $d \geq 2$. Set $m=\left[H+\frac{1}{2}\right]$ and $\beta_{H}=d-2(H-m)$. Then

$$
B_{H}(\varphi) \stackrel{f d d}{=} W_{\beta_{H}}\left((-\Delta)^{-m / 2} \varphi\right), \quad \varphi \in \mathcal{S}_{\infty}\left(\mathbb{R}^{d}\right) .
$$

Moreover, let $F$ be a $\sigma$-finite non-negative measure on $\mathbb{R}^{+}$satisfying $\mathbf{A}\left(\beta_{H}\right)$. For all positive functions $\lambda$ such that $\lambda(\rho) \rho^{\beta_{H}} \underset{\rho \rightarrow 0^{m}-H}{\longrightarrow}+\infty$, the limit

$$
\frac{X_{\rho}\left((-\Delta)^{-\frac{m}{2}} \varphi\right)-\mathbb{E}\left(X_{\rho}\left((-\Delta)^{-\frac{m}{2}} \varphi\right)\right)}{\sqrt{\lambda(\rho) \rho^{\beta_{H}}}} \underset{\rho \rightarrow 0^{m-H}}{\stackrel{f d d}{\longrightarrow}} B_{H}(\varphi)
$$

holds for all $\varphi \in \mathcal{S}_{\infty}\left(\mathbb{R}^{d}\right)$, in the sense of finite dimensional distributions of the random functionals.

For the case $H>-1 / 2$ the covariance functional of $B_{H}$ has the representation

$$
\operatorname{Cov}\left(B_{H}(\varphi), B_{H}(\psi)\right)=c_{H} \int_{\mathbb{R}^{d} \times \mathbb{R}^{d}}\left|z-z^{\prime}\right|^{2 H} \varphi(z) \psi\left(z^{\prime}\right) \mathrm{d} z \mathrm{~d} z^{\prime}, \quad \varphi, \psi \in \mathcal{S}_{\infty}\left(\mathbb{R}^{d}\right)
$$

Proof. According to Proposition 3.5, since $\beta_{H} \in(d-1, d+1) \subset(d-1,2 d)$ for $d=1$ and $\beta_{H} \in(d-1, d+1] \subset(d-1,2 d)$ for $d \geq 2$ with $\beta_{H} \neq d$, the random field $W_{\beta_{H}}$ is well-defined on $\mathcal{S}_{\infty}\left(\mathbb{R}^{d}\right)$. Moreover, for any $\varphi, \psi \in \mathcal{S}_{\infty}\left(\mathbb{R}^{d}\right)$, we have

$$
\begin{aligned}
& \left.\operatorname{Cov}\left(W_{\beta_{H}}\left((-\Delta)^{-m / 2} \varphi\right)\right), W_{\beta_{H}}\left((-\Delta)^{-m / 2} \psi\right)\right) \\
= & c_{\beta_{H}} \int_{\mathbb{R}^{d} \times \mathbb{R}^{d}}\left|z-z^{\prime}\right|^{d-\beta_{H}}(-\Delta)^{-m / 2} \varphi(z)(-\Delta)^{-m / 2} \psi\left(z^{\prime}\right) \mathrm{d} z \mathrm{~d} z^{\prime} \\
= & k_{\beta_{H}} \int_{\mathbb{R}^{d}}\left(-\widehat{\Delta)^{-m}} / 2 \varphi(\xi) \widehat{\left(-\widehat{\Delta)^{-m} / 2} \psi\right.}(\xi)|\xi|^{\beta_{H}-2 d} \mathrm{~d} \xi .\right.
\end{aligned}
$$


By (32) and (30) with $k_{H}=k_{\beta_{H}}$, we get

$$
\begin{aligned}
\left.\operatorname{Cov}\left(W_{\beta_{H}}\left((-\Delta)^{-m / 2} \varphi\right)\right), W_{\beta_{H}}\left((-\Delta)^{-m / 2} \psi\right)\right) & =k_{\beta_{H}} \int_{\mathbb{R}^{d}} \widehat{\varphi}(\xi) \bar{\psi}(\xi)|\xi|^{\beta_{H}-2 d-2 m} \mathrm{~d} \xi \\
& =\operatorname{Cov}\left(B_{H}(\varphi), B_{H}(\psi)\right) .
\end{aligned}
$$

Since the two random fields $W_{\beta_{H}}$ and $B_{H}$ are Gaussian this is enough to conclude that

$$
B_{H}(\varphi) \stackrel{\text { f.d.d. }}{=} W_{\beta_{H}}\left((-\Delta)^{-m / 2} \varphi\right) .
$$

Next, by rewriting (31),

$$
\operatorname{Cov}\left(B_{H}(\varphi), B_{H}(\psi)\right)=c_{\beta_{H}} \int_{\mathbb{R}^{d}}|z|^{d-\beta_{H}}\left((-\Delta)^{-m / 2} \varphi *(-\Delta)^{-m / 2} \psi\right)(z) \mathrm{d} z
$$

with

$$
(-\Delta)^{-m / 2} \varphi *(-\Delta)^{-m / 2} \psi(z)=\int_{\mathbb{R}^{d}}(-\Delta)^{-m / 2} \varphi\left(z-z^{\prime}\right)(-\Delta)^{-m / 2} \psi\left(z^{\prime}\right) \mathrm{d} z^{\prime} .
$$

Using Fourier transforms,

$$
(-\Delta)^{-m / 2} \varphi *(-\Delta)^{-m / 2} \psi(z)=(-\Delta)^{-m}(\varphi * \psi(z))
$$

so that

$$
\operatorname{Cov}\left(B_{H}(\varphi), B_{H}(\psi)\right)=c_{\beta_{H}} \int_{\mathbb{R}^{d}}|z|^{d-\beta_{H}}(-\Delta)^{-m}(\varphi * \psi)(z) \mathrm{d} z .
$$

Here, since $\Delta|z|^{2 H}=2 H(2(H-1)+d)|z|^{2 H-2}$, one can find a constant $c_{H, m}$ such that $|z|^{d-\beta_{H}}=|z|^{2 H-m}=c_{H, m} \Delta^{m}|z|^{2 H}$, for any $m \geq 0$. Then, for any $H>-\frac{1}{2}$, integrating by parts, we obtain

$$
\int_{\mathbb{R}^{d}}|z|^{d-\beta_{H}}(-\Delta)^{-m}(\varphi * \psi(z)) \mathrm{d} z=c_{H, m} \int_{\mathbb{R}^{d}}|z|^{2 H} \Delta^{m}\left((-\Delta)^{-m}(\varphi * \psi(z))\right) \mathrm{d} z .
$$

Thus,

$$
\operatorname{Cov}\left(B_{H}(\varphi), B_{H}(\psi)\right)=c_{H} \int_{\mathbb{R}^{d} \times \mathbb{R}^{d}}\left|z-z^{\prime}\right|^{2 H} \varphi(z) \psi\left(z^{\prime}\right) \mathrm{d} z \mathrm{~d} z^{\prime}
$$

with $c_{H}=(-1)^{m} c_{H, m} c_{\beta_{H}}$.

Finally, the last statement follows from Theorem 2.1.

Under the same parameter assumptions as in the previous theorem we may define analogously a continuous generalized random field $P_{H}$ on $\mathcal{S}_{\infty}\left(\mathbb{R}^{d}\right)$ by

$$
P_{H}(\varphi)=J_{\beta_{H}}\left((-\Delta)^{-m / 2} \varphi\right), \quad \varphi \in \mathcal{S}_{\infty}\left(\mathbb{R}^{d}\right) .
$$

The effect of a dilation by $a>0$, is given by

$$
J_{\beta_{H}}\left(\left((-\Delta)^{-m / 2} \varphi\right)_{a}\right)=J_{\beta_{H}}\left(a^{m}(-\Delta)^{-m / 2}\left(\varphi_{a}\right)\right)=a^{m} P_{H}\left(\varphi_{a}\right) .
$$

This allows us to extend Theorem 2.5 to the case of a general index $H$. By Proposition 3.5, the covariance functional of $P_{H}$ coincides with that of $B_{H}$. 
Theorem 3.8. Take $H$ a real number, $H \notin \frac{1}{2} \mathbb{Z}$ for $d=1, H \notin \mathbb{Z}$ for $d \geq 2$. As above, let $m=\left[H+\frac{1}{2}\right]$ and $\beta_{H}=d-2(H-m)$. Let $F$ be a non-negative measure on $\mathbb{R}^{+}$ which satisfies $\mathbf{A}\left(\beta_{H}\right)$. For all positive functions $\lambda$ such that $\lambda(\rho) \rho^{\beta_{H}} \underset{\rho \rightarrow 0^{m-H}}{\longrightarrow} a^{2(H-m)}$, for some $a>0$, we have in the sense of finite-dimensional distributions of random functionals the scaling limit

$$
X_{\rho}\left((-\Delta)^{-\frac{m}{2}} \varphi\right)-\mathbb{E}\left(X_{\rho}\left((-\Delta)^{-\frac{m}{2}} \varphi\right)\right) \underset{\rho \rightarrow 0^{m}-H}{\stackrel{f d d}{\longrightarrow}} a^{m} P_{H}\left(\varphi_{a}\right),
$$

for all $\varphi \in \mathcal{S}_{\infty}\left(\mathbb{R}^{d}\right)$.

\section{Pointwise representation of the random fields $B_{H}$ and $P_{H}$}

In this section we will discuss the case of a positive self-similarity index, and assume henceforth $H>0$. Recall that the Gaussian field $B_{H}$ is defined on $\mathcal{S}_{\lceil H\rceil}\left(\mathbb{R}^{d}\right.$ ) (since in this case $\lceil H\rceil_{+}=\lceil H\rceil$ ). By Proposition 3.4,

$$
\mathcal{S}_{\lceil H\rceil}\left(\mathbb{R}^{d}\right)=\operatorname{Span}\left\{D^{j} \varphi: \varphi \in \mathcal{S}\left(\mathbb{R}^{d}\right), j \in \mathbb{N}^{d},|j|=\lceil H\rceil\right\} .
$$

A natural question that arises in this context is whether it is possible to find a continuous random linear functional $Y$ on $\mathcal{S}\left(\mathbb{R}^{d}\right)$ such that

$$
\forall \varphi \in \mathcal{S}\left(\mathbb{R}^{d}\right), \quad D^{j} Y(\varphi)=(-1)^{|j|} B_{H}\left(D^{j} \varphi\right), \quad j \in \mathbb{N}^{d} \text { with }|j|=\lceil H\rceil ?
$$

The same question applies to the Poisson field $P_{H}$ defined by (33). We will use the representation of generalized random fields as defined by Matheron [16], to provide an answer (see also the links between "generalized random fields" and "punctual random fields" in [3]).

4.1. Representation of generalized random fields. Let $X$ be a continuous random linear functional on a subset $\mathcal{S}$ of $\mathcal{S}\left(\mathbb{R}^{d}\right)$. We say that a continuous function $\widetilde{X}: \mathbb{R}^{d} \rightarrow$ $L^{2}(\Omega, \mathcal{A}, \mathbb{P})$ is a representation of $X$ if, for any $\varphi \in \mathcal{S}$

$$
X(\varphi) \stackrel{L^{2}(\Omega, \mathcal{A}, \mathbb{P})}{=} \int_{\mathbb{R}^{d}} \tilde{X}(t) \varphi(t)(\mathrm{d} t) .
$$

In order to obtain representations $\widetilde{B}_{H}(t)$ of $B_{H}$ and $\widetilde{P}_{H}(t)$ of $P_{H}$, for any $t \in \mathbb{R}^{d}$, we will consider an approximation in $\mathcal{S}_{\infty}\left(\mathbb{R}^{d}\right)$ of the Dirac mass $\delta_{t}$ at $t$.

Following the same ideas as [16], let $\theta \in \mathcal{S}\left(\mathbb{R}^{d}\right)$ be a positive even function such that its Fourier transform $\hat{\theta}$ satisfies $\hat{\theta}(0)=\int_{\mathbb{R}^{d}} \theta(z) \mathrm{d} z=1$ and, for any $j \in \mathbb{N}^{d}, j \neq 0$,

$$
D^{j} \widehat{\theta}(0)=(-i)^{|j|} \int_{\mathbb{R}^{d}} z^{j} \theta(z) \mathrm{d} z=0 .
$$

It follows that, for any $k, l \in \mathbb{N}^{d}, \int_{\mathbb{R}^{d}} z^{k} D^{l} \theta(z) d z=i^{|k|} D^{k} \widehat{D^{l} \theta}(0)$. Since $\widehat{D^{l} \theta}(\xi)=$ $(i \xi)^{l} \widehat{\theta}(\xi)$, we obtain

$$
\int_{\mathbb{R}^{d}} z^{k} D^{l} \theta(z)(\mathrm{d} z)=\delta_{l}^{k}, \quad \delta_{l}^{k}= \begin{cases}0, & \text { if } k \neq l \\ 1, & \text { if } k=l .\end{cases}
$$


Let $n \in \mathbb{N}$ with $n \neq 0$ and set $\theta_{n}(z)=n^{d} \theta(n z)$. Then for all $t \in \mathbb{R}^{d}$, the functions defined by

$$
\Theta_{t}^{n}=\tau_{t} \theta_{n}-\sum_{|l|<\lceil H\rceil} \frac{(-1)^{|l|}}{l !} t^{l} D^{l} \theta_{n}
$$

belongs to $\mathcal{S}_{\infty}\left(\mathbb{R}^{d}\right) \subset \mathcal{S}_{\lceil H\rceil}\left(\mathbb{R}^{d}\right)$, and we can consider the sequence of random functions defined by $\left(B_{H}\left(\Theta_{\cdot}^{n}\right)\right)_{n \geq 1}$ and $\left(P_{H}\left(\Theta_{\cdot}^{n}\right)\right)_{n \geq 1}$, where $B_{H}\left(\Theta^{n}\right): t \mapsto B_{H}\left(\Theta_{t}^{n}\right)$ for all $n \geq 1$ and similarly for $P_{H}\left(\Theta^{n}\right)$.

Theorem 4.1. Let $H>0$ with $H \notin \mathbb{N}$. The finite dimensional distributions of $\left(B_{H}\left(\Theta^{n}\right)\right)_{n \geq 1}$ converge in $L^{2}(\Omega, \mathcal{A}, \mathbb{P})$ to a representation $\widetilde{B_{H}}$ of $B_{H}$ on $\mathcal{S}_{\lceil H\rceil}\left(\mathbb{R}^{d}\right)$ with a covariance function given by

$$
\begin{aligned}
& \Gamma_{H}(t, s)=k_{H} \int_{\mathbb{R}^{d}}\left(e^{-i t \cdot \xi}-\sum_{0 \leq k<\lceil H\rceil} \frac{(i t \cdot \xi)^{k}}{k !}\right) \overline{\left(e^{-i s \cdot \xi}-\sum_{0 \leq k<\lceil H\rceil} \frac{(i s \cdot \xi) k}{k !}\right)}|\xi|^{-d-2 H} \mathrm{~d} \xi \\
& \quad=c_{H}\left(|t-s|^{2 H}-\sum_{|l|<\lceil H\rceil} \frac{(-1)^{|l|}}{l !}\left(s^{l} D^{l}|t|^{2 H}+t^{l} D^{l}|s|^{2 H}\right)\right)
\end{aligned}
$$

Similarly, the finite dimensional distributions of $\left(P_{H}\left(\Theta^{n}{ }^{n}\right)\right)_{n \geq 1}$ converge in $L^{2}(\Omega, \mathcal{A}, \mathbb{P})$ to a representation $\widetilde{P_{H}}$ of $P_{H}$ on $\mathcal{S}_{\lceil H\rceil}\left(\mathbb{R}^{d}\right)$ with the same covariance function as $\widetilde{B_{H}}$.

Proof. Let $n \in \mathbb{N} \backslash\{0\}$ and $t \in \mathbb{R}^{d}$. By choice of $\theta$ we have $\Theta_{n}^{t} \in \mathcal{S}_{\infty}\left(\mathbb{R}^{d}\right)$, with

$$
\widehat{\Theta_{t}^{n}}(\xi)=\widehat{\theta}_{n}(\xi)\left(e^{-i t \cdot \xi}-\sum_{|l|<\lceil H\rceil} \frac{1}{l !} t^{l}(-i \xi)^{l}\right)=\widehat{\theta}\left(\frac{\xi}{n}\right)\left(e^{-i t \cdot \xi}-\sum_{k<\lceil H\rceil} \frac{(i t \cdot \xi)^{k}}{k !}\right), \quad \xi \in \mathbb{R}^{d},
$$

using the fact

$$
\sum_{|l|=k} \frac{1}{l !} t^{l}(-i \xi)^{l}=\frac{(i t \cdot \xi)^{k}}{k !}, \quad k \in \mathbb{N}
$$

which is a generalization of the binomial theorem. Let $n, m \in \mathbb{N} \backslash\{0\}$ and $t, s \in \mathbb{R}^{d}$. By (30) there exists $k_{H}>0$ such that the covariance

$$
\Gamma_{n, m}(t, s)=\operatorname{Cov}\left(B_{H}\left(\Theta_{t}^{n}\right), B_{H}\left(\Theta_{s}^{m}\right)\right)=\operatorname{Cov}\left(P_{H}\left(\Theta_{t}^{n}\right), P_{H}\left(\Theta_{s}^{m}\right)\right)
$$

can be written

$$
\Gamma_{n, m}(t, s)=k_{H} \int_{\mathbb{R}^{d}} \widehat{\Theta_{t}^{n}}(\xi) \widehat{\widehat{\Theta_{s}^{m}}}(\xi)|\xi|^{-2 H-d} \mathrm{~d} \xi
$$

Then Lebesgue's theorem implies that the limit in $\Gamma_{n, m}(t, s) \underset{n, m \rightarrow+\infty}{\longrightarrow} \Gamma_{H}(t, s)$, is given by

$$
\Gamma_{H}(t, s)=C_{H} \int_{\mathbb{R}^{d}}\left(e^{-i t \cdot \xi}-\sum_{k<\lceil H\rceil} \frac{(i t \cdot \xi)^{k}}{k !}\right) \overline{\left(e^{-i s \cdot \xi}-\sum_{k<\lceil H\rceil} \frac{(i s \cdot \xi)^{k}}{k !}\right)}|\xi|^{-2 H-d} \mathrm{~d} \xi .
$$


Therefore, the finite dimensional distributions of $\left(B_{H}\left(\Theta^{n}\right)\right)_{n \geq 1}$, converge in $L^{2}(\Omega, \mathcal{A}, \mathbb{P})$ to a centered random field $\widetilde{B_{H}}$. The finite dimensional distributions of $\left(P_{H}\left(\Theta^{n}\right)\right)_{n \geq 1}$ converge similarly to a limit $\widetilde{P_{H}}$. Both limit fields have the covariance function $\Gamma_{H}$.

Let us prove that $\widetilde{B_{H}}$ is a representation of $B_{H}$ on $\mathcal{S}_{\lceil H\rceil}\left(\mathbb{R}^{d}\right)$. The covariance function $\Gamma_{H}$ of $\widetilde{B_{H}}$ is continuous with respect to each variable and so $\widetilde{B_{H}}: \mathbb{R}^{d} \rightarrow L^{2}(\Omega, \mathcal{A}, \mathbb{P})$ is continuous. Then, the random linear functional $X: \varphi \in \mathcal{S}\left(\mathbb{R}^{d}\right) \mapsto \int_{\mathbb{R}^{d}} \widetilde{B_{H}}(t) \varphi(t)(\mathrm{d} t)$ is well defined since

$$
\operatorname{Var}(X(\varphi))=\int_{\mathbb{R}^{d}} \int_{\mathbb{R}^{d}} \operatorname{Cov}\left(\widetilde{B_{H}}(t), \widetilde{B_{H}}(s)\right) \varphi(t) \varphi(s) \mathrm{d} t \mathrm{~d} s<+\infty,
$$

using the fact that $\operatorname{Var}\left(\widetilde{B_{H}}\right)(t) \leq C|t|^{2 H}$. Finally, for any $\varphi \in \mathcal{S}_{\lceil H\rceil}\left(\mathbb{R}^{d}\right)$ we have $\operatorname{Var}(X(\varphi))=\operatorname{Var}\left(B_{H}(\varphi)\right)$ by Proposition 3.5, since $\int_{\mathbb{R}^{d}} t^{l} \varphi(t)(\mathrm{d} t)=0$ for $|l|<\lceil H\rceil$, which proves that $\widetilde{B_{H}}$ is a representation of $B_{H}$ on $\mathcal{S}_{\lceil H\rceil}\left(\mathbb{R}^{d}\right)$. The same arguments hold to prove that $\widetilde{P_{H}}$ is a representation of $P_{H}$ on $\mathcal{S}_{\lceil H\rceil}\left(\mathbb{R}^{d}\right)$.

It remains to establish (34). By Theorem 3.7, for all $n, m \in \mathbb{N} \backslash\{0\}$,

$$
\Gamma_{n, m}(t, s)=c_{H} \int_{\mathbb{R}^{d} \times \mathbb{R}^{d}}\left|z-z^{\prime}\right|^{2 H} \Theta_{t}^{n}(z) \Theta_{s}^{m}\left(z^{\prime}\right) \mathrm{d} z \mathrm{~d} z^{\prime} .
$$

For any $z^{\prime} \in \mathbb{R}^{d}$, the function $f_{z^{\prime}}(z)=\left|z-z^{\prime}\right|^{2 H}$ admits continuous derivatives of order $l$ on $\mathbb{R}^{d}$ for any $|l|<\lceil H\rceil$. Therefore, for any $z^{\prime} \in \mathbb{R}^{d}$,

$$
\begin{aligned}
& \int_{\mathbb{R}^{d}}\left|z-z^{\prime}\right|^{2 H} \Theta_{t}^{n}(z) \mathrm{d} z=f_{z^{\prime}} * \theta_{n}(t)-\sum_{|l|<\lceil H\rceil} \frac{t^{l}}{l !} D^{l} f_{z^{\prime}} * \theta_{n}(0) \\
& \underset{n \rightarrow+\infty}{\longrightarrow}\left|t-z^{\prime}\right|^{2 H}-\sum_{|l|<\lceil H\rceil} \frac{(-1)^{|l|}}{l !} t^{l} D^{l}\left|z^{\prime}\right|^{2 H} .
\end{aligned}
$$

By Lebesgue's theorem, as $n \rightarrow+\infty$,

$$
\lim _{n \rightarrow+\infty} \Gamma_{n, m}(t, s)=c_{H} \int_{\mathbb{R}^{d}}\left(\left|t-z^{\prime}\right|^{2 H}-\sum_{|l|<\lceil H\rceil} \frac{(-1)^{|l|}}{l !} t^{l} D^{l}\left|z^{\prime}\right|^{2 H}\right) \Theta_{s}^{m}\left(z^{\prime}\right) \mathrm{d} z^{\prime} .
$$

As previously we obtain

$$
\int_{\mathbb{R}^{d}}\left|t-z^{\prime}\right|^{2 H} \Theta_{s}^{m}\left(z^{\prime}\right) \mathrm{d} z^{\prime} \underset{m \rightarrow+\infty}{\longrightarrow}|t-s|^{2 H}-\sum_{|l|<\lceil H\rceil}(-1)^{|l|} \frac{s^{l}}{l !} D^{l}|t|^{2 H},
$$

while

$$
\int_{\mathbb{R}^{d}} D^{l}\left|z^{\prime}\right|^{2 H} \Theta_{s}^{m}\left(z^{\prime}\right) \mathrm{d} z^{\prime} \underset{m \rightarrow+\infty}{\longrightarrow} D^{l}|s|^{2 H} .
$$

Therefore $\Gamma_{H}(t, s)=\lim _{n, m \rightarrow+\infty} \Gamma_{n, m}(t, s)$ is also equal to (34).

Remark 4.2. In the case $H<0$ one can not find any representation of neither $B_{H}$ nor $P_{H}$ on $\mathcal{S}\left(\mathbb{R}^{d}\right)$. This is due to the fact that the variance of a random field which is second order self-similar of order $H<0$ is not bounded around 0 . 
Since $B_{H}$ is Gaussian, $\widetilde{B_{H}}$ is also Gaussian as a limit in $L^{2}(\Omega, \mathcal{A}, \mathbb{P})$ of a Gaussian functional. The spectral representation of $\widetilde{B_{H}}$ is given by

$$
\widetilde{B_{H}}(t) \stackrel{f d d}{=} \sqrt{k_{H}} \int_{\mathbb{R}^{d}}\left(e^{-i t \cdot \xi}-\sum_{k<\lceil H\rceil} \frac{(-i t \cdot \xi)^{k}}{k !}\right)|\xi|^{-H-d / 2} W(\mathrm{~d} \xi),
$$

where $W$ is the complex Brownian measure. This field is called elliptic Gaussian selfsimilar random field in [2].

4.2. Properties of the pointwise representation. One can define the $\lceil H\rceil$ th increments of $\widetilde{B_{H}}$ with lag $h \in \mathbb{R}^{d}$, which correspond to the discrete differentiation of order $\lceil H\rceil$, by

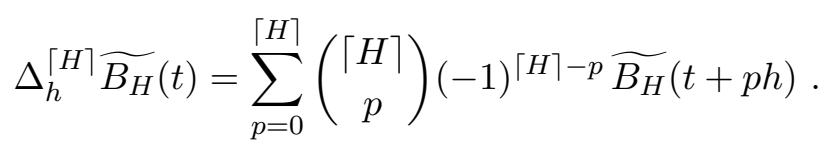

Then

$$
\Delta_{h}^{\lceil H\rceil} \widetilde{B_{H}}(t)=\lim _{n \rightarrow+\infty} B_{H}\left(\sum_{p=0}^{\lceil H\rceil}\left(\begin{array}{c}
\lceil H\rceil \\
p
\end{array}\right)(-1)^{\lceil H\rceil-p} \tau_{t+p h} \theta_{n}\right)
$$

and the stationarity of $B_{H}$ implies that $\widetilde{B_{H}}$ has stationary $\lceil H\rceil$ th increments in the wide sense: for all $t, s, h, h^{\prime} \in \mathbb{R}^{d}$, the covariances $\operatorname{Cov}\left(\Delta_{h}^{\lceil H\rceil} \widetilde{B_{H}}(s), \Delta_{h^{\prime}}^{\lceil H\rceil} \widetilde{B_{H}}(s+t)\right)$ do not depend on $s$ (see [22] or [10] for instance).

Proposition 4.3. Let $H>0$ with $H \notin \mathbb{N}$. Then the Gaussian random field $\widetilde{B_{H}}$ has stationary $\lceil H\rceil$ th increments. Moreover, this field admits continuous partial derivatives of order $l \in \mathbb{N}^{d}$ in mean square for any $|l|<\lceil H\rceil$, such that $D^{l} \bar{B}_{H}$ has stationary $(\lceil H\rceil-|l|)$ increments, is self-similar of order $\lceil H\rceil-|l|$, and satisfies $D^{l} \widetilde{B_{H}}(0)=0$ almost surely.

Proof. Recall that $\Gamma_{H}$ denotes the covariance function of $\widetilde{B_{H}}$. Since $\lceil H\rceil \geq 1$, it is straightforward to see that $\Gamma_{H}$ admits symmetric partial derivatives of order $l \in \mathbb{N}^{d}$ for any $|l|<\lceil H\rceil$, with $\frac{\partial^{2 l} \Gamma_{H}}{\partial s^{l} \partial t^{l}}(s, t)$ given by

$$
C_{H} \int_{\mathbb{R}^{d}}\left(e^{-i t \cdot \xi}-\sum_{k<\lceil H\rceil-|l|} \frac{(i t \cdot \xi)^{k}}{k !}\right) \overline{\left(e^{-i s \cdot \xi}-\sum_{k<\lceil H\rceil-|l|} \frac{(i s \cdot \xi)^{k}}{k !}\right)} \xi^{2 l}|\xi|^{-d-2 H} \mathrm{~d} \xi .
$$

By Theorem 2.2.2 of [1], this means that $\widetilde{B_{H}}$ admits a continuous partial derivative of order $l$ in mean square, $D^{l} \widetilde{B_{H}}$, which is a Gaussian random field with covariance given by $\operatorname{Cov}\left(D^{l} \widetilde{B_{H}}(t), D^{l} \widetilde{B_{H}}(s)\right)=\frac{\partial^{2 l} \Gamma_{H}}{\partial s^{l} \partial t^{l}}(s, t)$. A straightforward change of variables yields for all $a>0$

$$
\operatorname{Cov}\left(D^{l} \widetilde{B_{H}}(a t), D^{l} \widetilde{B_{H}}(a s)\right)=a^{2(H-l)} \operatorname{Cov}\left(D^{l} \widetilde{B_{H}}(t), D^{l} \widetilde{B_{H}}(s)\right) .
$$

Since $D^{l} \widetilde{B_{H}}$ is Gaussian this implies that $D^{l} \widetilde{B_{H}}$ is self-similar of order $H-|l|$, that is

$$
\left\{D^{l} \widetilde{B_{H}}(a t), t \in \mathbb{R}^{d}\right\} \stackrel{f d d}{=} a^{H-|l|}\left\{D^{l} \widetilde{B_{H}}(t), t \in \mathbb{R}^{d}\right\} \text { for all } a>0 .
$$


Moreover, for all $t, s, h, h^{\prime} \in \mathbb{R}^{d}$,

$$
\begin{aligned}
\operatorname{Cov} & \left(\Delta_{h}^{\lceil H\rceil-|l|} D^{l} \widetilde{B_{H}}(s), \Delta_{h^{\prime}}^{\lceil H\rceil-|l|} D^{l} \widetilde{B_{H}}(s+t)\right) \\
& =C_{H} \int_{\mathbb{R}^{d}} e^{-i t \cdot \xi}\left(e^{-i h \cdot \xi}-1\right)^{\lceil H\rceil-|l|}\left(e^{i h^{\prime} \cdot \xi}-1\right)^{\lceil H\rceil-|l|} \xi^{2 l}|\xi|^{-2 H-d} \mathrm{~d} \xi,
\end{aligned}
$$

and $D^{l} \widetilde{B_{H}}$ has stationary $(\lceil H\rceil-|l|)$ th increments. Finally, $\operatorname{Var}\left(D^{l} \widetilde{B_{H}}(0)\right)=0$ implies that $D^{l} \widetilde{B_{H}}(0)=0$ almost surely.

Specializing to the case $d=1$, the covariance function $\Gamma_{H}$ in (34) equals

$$
c_{H}\left(|t-s|^{2 H}-\sum_{l<\lceil H\rceil}(-1)^{l}\left(\begin{array}{c}
2 H \\
l
\end{array}\right)\left(\left(\frac{s}{t}\right)^{l}|t|^{2 H}+\left(\frac{t}{s}\right)^{l}|s|^{2 H}\right)\right),
$$

where $\left(\begin{array}{c}2 H \\ l\end{array}\right)=(2 H) \cdot \ldots \cdot(2 H-(l-1)) / l$ !. Therefore $\sqrt{\frac{C_{H}}{2(-1)^{|H|} c_{H}}} \widetilde{B_{H}}$ is a $\lceil H\rceil$ th-order fractional Brownian motion as defined in [18], with $C_{H}^{-1}=\Gamma(1+2 H)|\sin (H \pi)|$.

\section{Remark 4.4.}

a) One can prove that $\widetilde{B_{H}}$ is the only Gaussian random field with stationary $\lceil H\rceil$ th increments, which is self-similar of order $\lceil H\rceil$ and isotropic.

b) The representation $\widetilde{P_{H}}$ of $P_{H}$ obtained in Theorem 4.1 is not Gaussian but shares the same covariance function as $\widetilde{B_{H}}$. Therefore it satisfies the same second order properties: stationary $\lceil H\rceil$ th increments, self-similarity of order $H$ and isotropy.

4.3. Fractional Brownian field and fractional Poisson field. For $0<H<1$, the random field $\widetilde{B_{H}}$ corresponds to the well known fractional Brownian field with Hurst parameter equal to $H$ and (35) is known as the harmonizable representation of the fractional Brownian field (see [11] for a review).

We consider the special case $0<H<1 / 2$ for which $d-1<\beta_{H}=d-2 H<d$. For this range of parameters, $\lceil H\rceil=1$ and

$$
\widetilde{\mathcal{M}}_{\beta_{H}}=\mathcal{M}^{\beta_{H}} \cap \mathcal{M}_{1}, \quad \mathcal{M}_{1}=\left\{\mu \in \mathcal{M}: \int_{\mathbb{R}^{d}} \mu(d z)=0\right\} .
$$

It follows that all pointwise increment measures $\delta_{x}-\delta_{0}, x \in \mathbb{R}^{d}$, belong to $\widetilde{\mathcal{M}}_{\beta_{H}}$, and are hence admissible for evaluating the limit fields $W_{\beta_{H}}$ and $J_{\beta_{H}}$. Using the representations $\widetilde{B_{H}}$ and $\widetilde{P_{H}}$ in Theorem 4.1 it is verified that $\widetilde{B_{H}}(x) \stackrel{f d d}{=} W_{\beta_{H}}\left(\delta_{x}-\delta_{0}\right)$ and $\widetilde{P_{H}}(x) \stackrel{f d d}{=}$ $J_{\beta_{H}}\left(\delta_{x}-\delta_{0}\right)$.

To analyze the properties of $\widetilde{P_{H}}$ we observe using (24),

$$
\log \mathbb{E}\left(\exp \left(i \widetilde{P_{H}}(x)\right)\right)=\int_{\mathbb{R}^{+} \times \mathbb{R}^{d}} \Psi\left(\delta_{x}(B(y, r))-\delta_{0}(B(y, r))\right) C_{\beta_{H}} \mathrm{~d} y r^{-\beta_{H}-1} \mathrm{~d} r
$$


Here,

$$
\delta_{x}(B(y, r))-\delta_{0}(B(y, r))=\left\{\begin{array}{cl}
1, & |x-y|<r<|y| \\
-1, & |y|<r<|x-y| \\
0 & \text { otherwise }
\end{array}\right.
$$

and hence we may recast (36) into

$$
\begin{aligned}
\log \mathbb{E}\left(\exp \left(i \theta \widetilde{P_{H}}(x)\right)\right)= & \Psi(\theta) \int_{\mathbb{R}^{+} \times \mathbb{R}^{d}} \mathbf{1}_{\{|x-y|<r<|y|\}} C_{\beta_{H}} \mathrm{~d} y r^{-\beta_{H}-1} \mathrm{~d} r \\
& +\Psi(-\theta)) \int_{\mathbb{R}^{+} \times \mathbb{R}^{d}} \mathbf{1}_{\{|y|<r<|x-y|\}} C_{\beta_{H}} \mathrm{~d} y r^{-\beta_{H}-1} \mathrm{~d} r \\
= & \left(-c_{H}\right)|x|^{2 H}(\Psi(\theta)+\Psi(-\theta)) .
\end{aligned}
$$

This is the logarithmic characteristic functional of the difference of two independent random variables both having a Poisson distribution with intensity $\left(-c_{H}\right)|x|^{2 H}$. Hence, $\widetilde{P}_{H}(x), x \in \mathbb{R}^{d}$, defines a mean zero integer-valued symmetrized Poisson distributed random field, such that for any $x, x^{\prime} \in \mathbb{R}^{d}$,

$$
\operatorname{Cov}\left(\widetilde{P_{H}}(x), \widetilde{P_{H}}\left(x^{\prime}\right)\right)=\left(-c_{\beta}\right)\left(|x|^{2 H}+\left|x^{\prime}\right|^{2 H}-\left|x-x^{\prime}\right|^{2 H}\right) .
$$

By analogy with fractional Brownian field this makes it natural to view $\widetilde{P_{H}}$ as a fractional Poisson field.

Some remarks are in order concerning the fractional fields obtained here in relation to so called Chentsov random fields, in particular Takenaka fields, see [21], [20] Ch. 8. By (36),

$$
\widetilde{P_{H}}(x) \stackrel{f d d}{=} \int_{\mathbb{R}^{d} \times \mathbb{R}^{+}}\left(\mathbf{1}_{B(x, r)}(y)-\mathbf{1}_{B(0, r)}(y)\right) \widetilde{N}_{\beta_{H}}(\mathrm{~d} y, \mathrm{~d} r),
$$

where $\widetilde{N}_{\beta_{H}}$ is a compensated Poisson random measure with intensity $C_{\beta_{H}} r^{-\beta_{H}-1} \mathrm{~d} r \mathrm{~d} y$. Let us assume, for the sake of this discussion, that we fix a parameter $1<\alpha<2$ and apply random weights $w$ symmetrically with intensity $\nu(d w)=C_{\alpha}|w|^{-(1+\alpha)}$ to the points $(y, r)$ of the Poisson measure, and hence replaces $\widetilde{N}_{\beta_{H}}$ with a measure $w \widetilde{N}_{\beta_{H}}(d y, d r, d w)$. The resulting field

$$
Y(x)=\int_{\mathbb{R}^{d} \times \mathbb{R}^{+} \times \mathbb{R}}\left(\mathbf{1}_{B(x, r)}(y)-\mathbf{1}_{B(0, r)}(y)\right) w \tilde{N}_{\beta_{H}}(\mathrm{~d} y, \mathrm{~d} r, \mathrm{~d} w)
$$

has the representation

$$
Y(x) \stackrel{f d d}{=} \int_{\mathbb{R}^{d} \times \mathbb{R}^{d}}\left(\mathbf{1}_{B(x, r)}(y)-\mathbf{1}_{B(0, r)}(y)\right) M_{\alpha}(\mathrm{d} y, \mathrm{~d} r)
$$

where $M_{\alpha}$ is an $\alpha$-stable random measure with associated measure proportional to $r^{-\beta_{H}-1} \mathrm{~d} r \mathrm{~d} y,[20] \mathrm{Thm}$. 3.12.2. By properties of stochastic integrals with respect to symmetric $\alpha$-stable measures we have, for some constant $C$,

$$
\begin{aligned}
\log \mathbb{E}(\exp (i \theta Y(x))) & =-C \int_{\mathbb{R}^{+} \times \mathbb{R}^{d}}|\theta|^{\alpha}\left|\mathbf{1}_{B(x, r)}(y)-\mathbf{1}_{B(0, r)}(y)\right|^{\alpha} \mathrm{d} y r^{-\beta_{H}-1} \mathrm{~d} r \\
& =-C|\theta|^{\alpha} \int_{\mathbb{R}^{+} \times \mathbb{R}^{d}} \mathbf{1}_{B(x, r) \Delta B(0, r)}(y) \mathrm{d} y r^{-\beta_{H}-1} \mathrm{~d} r
\end{aligned}
$$


where $\Delta$ denotes the symmetric set difference. Hence

$$
Y(x) \stackrel{f d d}{=} \int_{\mathbb{R}^{d} \times \mathbb{R}^{d}} \mathbf{1}_{B(x, r) \Delta B(0, r)}(y) M_{\alpha}(\mathrm{d} y, \mathrm{~d} r),
$$

which defines a symmetric $\alpha$-stable random field which is self-similar with index $H^{\prime}=$ $\left(d-\beta_{H}\right) / \alpha \in(0,1 / \alpha)$, known as a $\left(\alpha, H^{\prime}\right)$-Takenaka field, [20] Definition 8.4.1 (the parameter $\beta$ of the reference corresponds to $d-\beta_{H}$ in our notation). It is noticed in [20], moreover, that $\widetilde{B_{H}}$ is a $(2, H)$-Takenaka field. Kaj and Taqqu [14] (for the case $d=1$ ) consider random balls models with randomized weights and study limiting schemes in the Gaussian, Poisson and stable regimes, using in particular Poisson representations of stable random measures as above.

The fractional Poisson field $\widetilde{P_{H}}$ shares with $\widetilde{B_{H}}$ and with $(\alpha, H)$-Takenaka fields ([20] Thm 8.6.3) a well known interesting invariance property under restriction to lowerdimensional hyperplanes. For example, any cut along a line through a planar fractional field in $\mathbb{R}^{2}$ generates a one-dimensional fractional process of the same kind. To see this, let $H_{k}$ be a $k$-dimensional hyperplane in $\mathbb{R}^{d}$. We consider $\mathbb{R}^{d}=H_{k} \oplus H_{k}^{\perp}$ and write $\bar{x}_{k}$ for the restriction to $H_{k}$ of $x=\bar{x}_{k}+\left(x-\bar{x}_{k}\right) \in \mathbb{R}^{d}$. To emphasize the dimensional dependence we write here $\widetilde{B_{H, d}}(x)$ and $\widetilde{P_{H, d}}(x)$ respectively, if the fractional fields are defined on $\mathbb{R}^{d}$.

Proposition 4.5. Given $H \in(0,1 / 2)$, let $\beta_{H}^{\prime}=\beta_{H}-d+k \in(k-1, k)$. Then the measure $\delta_{\bar{x}_{k}}-\delta_{0}$ belongs to $\widetilde{\mathcal{M}}_{\beta_{H}^{\prime}}$, and we have

$$
\widetilde{B_{H, d}}\left(\bar{x}_{k}\right) \stackrel{f d d}{=} \widetilde{B_{H^{\prime}, k}}\left(\bar{x}_{k}\right)
$$

and

$$
\widetilde{P_{H, d}}\left(\bar{x}_{k}\right) \stackrel{f d d}{=} \widetilde{P_{H^{\prime}, k}}\left(\bar{x}_{k}\right)
$$

for $H^{\prime}=\frac{k-\beta_{H}^{\prime}}{2}=\frac{d-\beta_{H}}{2}=H$.

Proof. It is enough to consider hyperplanes of the form $x=\left(x_{1}, \ldots, x_{k}, 0, \ldots, 0\right)$. Then, clearly, $\left|\bar{x}_{k}\right|^{d-\beta_{H}}=\left|\bar{x}_{k}\right|^{k-\beta_{H}^{\prime}}$, which carries over to showing that the covariances of the pair of relevant random fields coincide.

\section{Aggregate similarity}

We have seen that the Gaussian limit field $W_{\beta}$ is self-similar whereas the Poisson limit $J_{\beta}$ is not. A similarity property which applies in great generality to long-range dependent processes is discussed in [12]. The following is a version for spatial random fields.

Definition 5.1. A random field $X$ with $\mathbb{E} X=0$, defined on a subspace $\mathcal{S}$ of $\mathcal{M}$ which is closed for dilations, is said to be aggregate-similar if there exists a sequence of positive real numbers $\left(a_{m}\right)_{m \geq 1}$, such that

$$
\forall \mu \in \mathcal{S}, \forall m \geq 1, X\left(\mu_{a_{m}}\right) \stackrel{f d d}{=} \sum_{i=1}^{m} X^{i}(\mu),
$$


where $\left(X^{i}\right)_{i \geq 1}$ are i.i.d copies of $X$.

Thus, a random field is aggregate-similar if the path $\mu_{a_{m}} \mapsto X\left(\mu_{a_{m}}\right)$ as we trace along the sequence of dilations given by $a_{m}$ passes all aggregates $\sum_{i=1}^{m} X^{i}$ of $X$, in the distributional sense. We may write, equivalently,

$$
\forall \mu \in \mathcal{S}, \forall m \geq 1, X(\mu) \stackrel{f d d}{=} \sum_{i=1}^{m} X^{i}\left(\mu_{a_{m}^{-1}}\right),
$$

which immediately shows that an aggregate-similar random field is also infinitely divisible.

Any self-similar, zero mean Gaussian random field is aggregate-similar. Indeed, if $X_{H}$ is Gaussian with $\mathbb{E} X_{H}=0$ and self-similar with index $H$ then letting $a_{m}=m^{1 / 2 H}$ we have

$$
X_{H}\left(\mu_{a_{m}}\right) \stackrel{f d d}{=} m^{1 / 2} X_{H}(\mu) \stackrel{f d d}{=} \sum_{i=1}^{m} X_{H}^{i}(\mu), \quad m \geq 1
$$

In particular, $W_{\beta}$ is aggregate-similar on $\widetilde{\mathcal{M}}_{\beta}$ with respect to the sequence $a_{m}=$ $m^{1 /(d-\beta)}$. For $d-1<\beta<d$ we have $a_{m}^{-1} \rightarrow 0$ and hence $\mu_{a_{m}}$ represents a zoomin of $W_{\beta}$, as $m \rightarrow \infty$. This is in contrast to the case $d<\beta<2 d$ for which $a_{m}^{-1} \rightarrow \infty$. Consequently, the succession of aggregates $\sum_{i=1}^{m} W_{\beta}^{i}(\mu)$ of $W_{\beta}(\mu)$ appear as the sequence of measures $\mu_{a_{m}}$ performs a zoom-out, in the limit $m \rightarrow \infty$. In agreement with (37), using again $a_{m}=m^{1 / 2 H}$, the random fields $B_{H}$ are aggregate-similar with respect to the subspace $\mathcal{S}_{\infty}$, that is

$$
B_{H}\left(\varphi_{a_{m}}\right) \stackrel{f d d}{=} \sum_{i=1}^{m} B_{H}^{i}(\varphi), \quad m \geq 1 .
$$

Turning next to the non-Gaussian field $J_{\beta}$, by (24)

$$
\log \mathbb{E}\left(\exp \left(i J_{\beta}\left(\mu_{a}\right)\right)\right)=a^{\beta-d} \log \mathbb{E}\left(\exp \left(i J_{\beta}(\mu)\right)\right) .
$$

Thus, $J_{\beta}$ is aggregate-similar with respect to $a_{m}$, given by $a_{m}^{\beta-d}=m$. The same applies to $P_{H}$ with $a_{m}^{2 H}=m$. This property provides an interpretation of the dilation parameter $a$ in Theorem 2.5. If we assume in the theorem that $\lambda(\rho) \rho^{\beta} \rightarrow a_{m}^{d-\beta}$ as $\rho^{\beta-d} \rightarrow 0$, for arbitrary $m \geq 1$, then

$$
X_{\rho}(\mu)-\mathbb{E}\left(X_{\rho}(\mu)\right) \stackrel{f d d}{\rightarrow} J_{\beta}\left(\mu_{a_{m}}\right) \stackrel{f d d}{=} \sum_{i=1}^{m} J_{\beta}^{i}(\mu)
$$

The guiding asymptotic quantity $\lambda \rho^{\beta}$ may be interpreted as the expected number of very large $(\beta>d)$ or very small $(\beta<d)$ balls which cover a point asymptotically. Thus, the more of such extreme grains are allowed asymptotically, the larger number of i.i.d. copies of the basic field $J_{\beta}$ appears in the limit. 
We may continue this line of reasoning by providing a limit result for $J_{\beta}\left(\mu_{a_{m}}\right)$ as $m \rightarrow \infty$. In view of Theorems 2.5 and 2.1 this result is not at all surprising.

Proposition 5.2. As $a^{d-\beta} \rightarrow \infty$, for all $\mu$ in $\widetilde{\mathcal{M}}_{\beta}$

$$
\frac{1}{a^{(d-\beta) / 2}} J_{\beta}\left(\mu_{a}\right) \stackrel{f d d}{\rightarrow} W_{\beta}(\mu)
$$

Proof. Consider the subsequence $a_{m}=m^{1 /(d-\beta)}$. It follows immediately from aggregatesimilarity and the central limit theorem that

$$
\frac{1}{a_{m}^{(d-\beta) / 2}} J_{\beta}\left(\mu_{a_{m}}\right) \stackrel{f d d}{=} \frac{1}{\sqrt{m}} \sum_{i=1}^{m} J_{\beta}^{i}(\mu) \stackrel{f d d}{\rightarrow} W_{\beta}(\mu), \quad m \rightarrow \infty,
$$

since $J_{\beta}(\mu)$ and $W_{\beta}(\mu)$ have the same variance. A standard argument completes the proof of convergence in distribution along an arbitrary sequence.

\section{REFERENCES}

[1] Adler, R. J. The Geometry of Random Field, John Wiley \& Sons, (1981).

[2] Benassi, A. and Jaffard, S. and Roux, D., Elliptic Gaussian random processes, Rev. Matem. Iberoamericana, 13, 1, 19-89, (1997).

[3] Biermé, H.: Champs aléatoires : autosimilarité, anisotropie et étude directionnelle, PhD report,

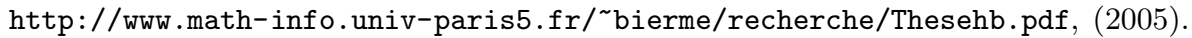

[4] Biermé, H. and Estrade A.: Poisson random balls: self-similarity and X-ray images, Adv. Appl. Prob., 38, 1-20, (2006).

[5] Biermé, H., Estrade A. and Kaj I.: About scaling behavior of random balls models, $S^{4} G$ th Int.

Conference, published by Union of Czech mathematicians and physicists, 63-68, (2006).

[6] Chi, Z.: Construction of stationary self-similar generalized fields by random wavelet expansion, Probab. Theory Relat. Fields, 121, 269-300, (2001).

[7] Dobrushin, R. L.: Gaussian and their subordinated self-similar random generalized fields, Ann. Probab., 7(1), 1-28, (1979).

[8] Guelfand, I. M. and Chilov, G. E. Les Distributions I, Dunod, (1962).

[9] Guelfand, I. M. and N. Y. Vilenkin, Les Distributions IV: Applications de l'Analyse Harmonique, Dunod, (1967).

[10] Guérin, C. A., Wavelet analysis and covariance structure of non-stationary processes, J. Fourier Anal. Appl., 6, 403-425, (2000).

[11] Herbin, E. From N-parameter fractional Brownian motions to N-parameter multifractional Brownian motion, Rocky Mountain J. of Math, 36, n4, 1249-1284, (2006).

[12] Kaj, I.: Limiting fractal random processes in heavy-tailed systems, In: Fractals in Engineering, New Trends in Theory and Applications, 199-218, Springer-Verlag London, (2005).

[13] Kaj, I., Leskelä, L., Norros, I. and Schmidt, V. Scaling limits for random fields with long-range dependence, Ann. Probab. 35, 528-550, (2007).

[14] Kaj, I. and Taqqu, M.S. Convergence to fractional Brownian motion and to the Telecom process: the integral representation approach. To appear: Brazilian Probability School, 10th anniversary volume, Eds. M.E. Vares, V. Sidoravicius, Birkhauser 2007.

[15] Kallenberg, O. Foundations of Modern Probability. Second edition, Springer-Verlag. (2002).

[16] Matheron, G. The intrinsic random functions and their applications, Adv. Appl. Prob., 5, 439-468, (1973).

[17] Medina, J. M. and Cernuschi-Frías, B., A synthesis of $1 / f$ process via Sobolev spaces and fractional integration, IEEE Trans. Inform. Theory, 51, n12, 4278-4285, (2005).

[18] Perrin, E., Harba, R., Berzin-Joseph, C., Iribarren, I. and Bonami, A., nth-order fractional Brownian motion and fractional Gaussian noises, IEEE Trans. Sign. Proc., 45, 1049-1059, (2001).

[19] Rudin, W. Real and complex analysis, Mc-Graw-Hill, (1966). 
[20] Samorodnitsky, G. and Taqqu, M. S. Stable non-Gaussian random processes, Chapman \& Hall, (1994).

[21] Takenaka, S. Integral-geometric construction of self-similar stable processes, Nagoya Math. J., 123, $1-12,(1991)$.

[22] Yaglom, A. M. Correlation Theory of Stationary and Related Random Functions (I), SpringerVerlag, (1997).

Hermine BIERMÉ, MAP5, Université Paris Descartes, 45 Rue des Saints-Pères, 75006 PARIS, FRANCE

E-mail address: hermine.bierme@math-info.univ-paris5.fr

Anne ESTrade, MAP5, Université Paris Descartes, 45 rue des Saints-Pères, 75006 PARIS, FRANCE

E-mail address: anne.estrade@univ-paris5.fr

Ingemar KAJ, Department of mathematics Uppsala University P.O. Box 480 S-751 06 , UPPSALA, SWEDEN

E-mail address: ikaj@math.uu.se 